\title{
Artin $L$-functions and modular forms associated to quasi-cyclotomic fields
}

\author{
by \\ Sunghan Bae (Daejeon), Yong Hu (Beijing), \\ and Linsheng Yin (Beijing)
}

1. Introduction. A quadratic extension of a cyclotomic field, which is non-abelian Galois over the rational number field $\mathbb{Q}$, is called a quasicyclotomic field. All quasi-cyclotomic fields are described explicitly in [8] following the work in [1] and [3]. Actually for any cyclotomic field $\mathbb{Q}\left(\zeta_{n}\right)$ we construct a canonical $\mathbb{Z} / 2 \mathbb{Z}$-basis of the quotient space of $\left\{\alpha \in \mathbb{Q}^{*} / \mathbb{Q}^{* 2}\right.$ $\mathbb{Q}\left(\zeta_{n}, \sqrt{\alpha}\right) / \mathbb{Q}$ is Galois $\}$ modulo the subspace $\left\{\alpha \in \mathbb{Q}^{*} / \mathbb{Q}^{* 2} \mid \mathbb{Q}\left(\zeta_{n}, \sqrt{\alpha}\right) / \mathbb{Q}\right.$ is abelian\}. The minimal quasi-cyclotomic field containing the square root of a special element of the basis is called a primary quasi-cyclotomic field. L. S. Yin and C. Zhang [7] have studied the arithmetic of any quasi-cyclotomic field. In this paper we determine all irreducible representations of primary quasi-cyclotomic fields. Our methods enable one to determine the irreducible representations of an arbitrary quasi-cyclotomic field. We also compute the Artin conductors of the representations and the Artin $L$-functions for a class of quasi-cyclotomic fields. They correspond to a series of normalized newforms of weight one by Deligne-Serre's theorem [6, Th. 2]. We describe these modular forms explicitly.

First we recall the construction of primary quasi-cyclotomic fields. Let $S$ be the set consisting of -1 and all prime numbers. For $p \in S$, we put $\bar{p}=4,8, p$ and set $p^{*}=-1,2,(-1)^{(p-1) / 2} p$ if $p=-1,2$ and an odd prime number, respectively. For prime numbers $p<q$, we define

$$
v_{p q}=\prod_{i=0}^{(p-1) / 2} \prod_{j=0}^{(q-1) / 2} \frac{\sin \frac{i q+j}{p q} \pi}{\sin \frac{j p+i}{p q} \pi} \quad((i, j) \neq(0,0), p>2)
$$

and

2010 Mathematics Subject Classification: 11R42, 11F30, 11F80, 11R21.

Key words and phrases: Galois representation, Artin $L$-function, modular form of weight one. 


$$
v_{2 q}=\frac{\sin \frac{\pi}{4}}{\sin \frac{\pi}{4 q}} \prod_{j=1}^{(q-1) / 2} \frac{\sin \frac{j \pi}{2 q} \cdot \sin \frac{2 j-1}{4 q} \pi}{2 \sin \frac{4 j+1}{4 q} \pi \cdot \sin \frac{j}{q} \pi \cdot \sin \frac{2 j-1}{2 q} \pi} .
$$

For $p<q \in S$, we put

$$
u_{p q}= \begin{cases}\sqrt{q^{*}} & \text { if } p=-1, \\ v_{p q} & \text { if } p=2 \text { or } p \equiv q \equiv 1 \bmod 4, \\ \sqrt{p} \cdot v_{p q} & \text { if } p \equiv 1, q \equiv 3 \bmod 4, \\ \sqrt{q} \cdot v_{p q} & \text { if } p \equiv 3, q \equiv 1 \bmod 4, \\ \sqrt{p q} \cdot v_{p q} & \text { if } p \equiv q \equiv 3 \bmod 4 .\end{cases}
$$

The canonical $\mathbb{Z} / 2 \mathbb{Z}$-basis of the quotient space mentioned above is a subset of $\left\{u_{p q} \mid p<q \in S\right\}$. For $p<q \in S$ let $K=\mathbb{Q}\left(\zeta_{\bar{p} q}\right)$ be the cyclotomic field of conductor $\bar{p} q$ and let $\widetilde{K}=K\left(\sqrt{u_{p q}}\right)$. Then $\widetilde{K}$ is the smallest quasicyclotomic fields containing $\sqrt{u_{p q}}$. We call these fields $\widetilde{K}$ primary quasicyclotomic fields. Let $G=\operatorname{Gal}(K / \mathbb{Q})$ and $\widetilde{G}=\operatorname{Gal}(\widetilde{K} / \mathbb{Q})$. We always denote by $\varepsilon$ the unique non-trivial element of $\operatorname{Gal}(\widetilde{K} / K)$. If $(p, q)=(-1,2)$, then the group $G$ is generated by two elements $\sigma_{-1}$ and $\sigma_{2}$, where $\sigma_{-1}\left(\zeta_{8}\right)=\zeta_{8}^{-1}$ and $\sigma_{2}\left(\zeta_{8}\right)=\zeta_{8}^{5}$. If $p=-1$ and $q \neq 2$, or if $p>2$, then $G$ is generated by two elements $\sigma_{p}$ and $\sigma_{q}$, where $\sigma_{p}\left(\zeta_{p}\right)=\zeta_{p}^{a}, \sigma_{p}\left(\zeta_{q}\right)=\zeta_{q}$ and $\sigma_{q}\left(\zeta_{p}\right)=\zeta_{p}$, $\sigma_{q}\left(\zeta_{q}\right)=\zeta_{q}^{b}$, with $a, b$ being generators of $(\mathbb{Z} / \bar{p} \mathbb{Z})^{*}$ and $(\mathbb{Z} / q \mathbb{Z})^{*}$ respectively. If $p=2$, then $G$ is generated by three elements $\sigma_{-1}, \sigma_{2}$ and $\sigma_{q}$, where $\sigma_{-1}$, $\sigma_{2}$ act on $\zeta_{8}$ as above and on $\zeta_{q}$ trivially, and $\sigma_{q}$ acts on $\zeta_{q}$ as above and on $\zeta_{8}$ trivially.

Next we describe the group $\widetilde{G}$ by generators and relations. An element $\sigma \in G$ has two lifts in $\widetilde{G}$. By [6, Sect. 3] the action of the two lifts on $\sqrt{u_{p q}}$ has the form $\pm \alpha \sqrt{u_{p q}}$ or $\pm \alpha \sqrt{u_{p q}} / \sqrt{-1}$ with $\alpha>0$. We fix the lift $\widetilde{\sigma}$ of $\sigma$ to be the one with a positive sign. Then the other lift of $\sigma$ is $\tilde{\sigma} \varepsilon$. The group $\widetilde{G}$ is generated by $\varepsilon, \widetilde{\sigma}_{p}$ and $\widetilde{\sigma}_{q}$ (and $\widetilde{\sigma}_{-1}$ if $p=2$ ). Clearly $\varepsilon$ commutes with the other generators. In addition, we have $\widetilde{\sigma}_{p} \widetilde{\sigma}_{q}=\widetilde{\sigma}_{q} \widetilde{\sigma}_{p} \varepsilon$ (and $\widetilde{\sigma}_{-1}$ commutes with $\widetilde{\sigma}_{2}$ and $\widetilde{\sigma}_{q}$ if $p=2$ ). For an element $g$ of a group, we denote by $|g|$ the order of $g$ in the group. Let $\log _{-1}:\{ \pm 1\} \rightarrow \mathbb{Z} / 2 \mathbb{Z}$ be the unique isomorphism. For an odd prime $p$ and an integer $a$ with $p \nmid a$, let $\left(\frac{a}{p}\right)$ be the quadratic residue symbol. We also define $\left(\frac{a}{2}\right)=\left(\frac{a}{-1}\right)=1$ for any $a$. Then we have (see [6, Th. 3])

$$
\left|\widetilde{\sigma}_{p}\right|=\left(1+\log _{-1}\left(\frac{q^{*}}{p}\right)\right)\left|\sigma_{p}\right| \text { and } \quad\left|\widetilde{\sigma}_{q}\right|=\left(1+\log _{-1}\left(\frac{p^{*}}{q}\right)\right)\left|\sigma_{q}\right|,
$$

with the exception that $\left|\widetilde{\sigma}_{2}\right|=2\left|\sigma_{2}\right|$ when $(p, q)=(-1,2)$. If $p=2$, we have furthermore $\left|\widetilde{\sigma}_{-1}\right|=\left|\sigma_{-1}\right|$. Thus we have completely determined the group $\widetilde{G}$ by generators and relations. 
2. Abelian subgroup of index 2. In this section we construct a special abelian subgroup of $\widetilde{G}$ of index 2 and determine its structure. We consider the following three cases separately:

Case A: $\left|\widetilde{\sigma}_{p}\right|=\left|\sigma_{p}\right|$ and $\left|\widetilde{\sigma}_{q}\right|=\left|\sigma_{q}\right|$;

Case B: $\left|\widetilde{\sigma}_{p}\right|=2\left|\sigma_{p}\right|,\left|\widetilde{\sigma}_{q}\right|=\left|\sigma_{q}\right|$ or $\left|\widetilde{\sigma}_{p}\right|=\left|\sigma_{p}\right|,\left|\widetilde{\sigma}_{q}\right|=2\left|\sigma_{q}\right|$;

Case C: $\left|\widetilde{\sigma}_{p}\right|=2\left|\sigma_{p}\right|$ and $\left|\widetilde{\sigma}_{q}\right|=2\left|\sigma_{q}\right|$.

All the three cases may happen: Case A if and only if $\left(\frac{p^{*}}{q}\right)=\left(\frac{q^{*}}{p}\right)=1$; Case B if and only if $\left(\frac{p^{*}}{q}\right) \neq\left(\frac{q^{*}}{p}\right)$ or $(p, q)=(-1,2)$; Case $\mathrm{C}$ if and only if $\left(\frac{p^{*}}{q}\right)=\left(\frac{q^{*}}{p}\right)=-1$.

In Case A, we define the subgroup $N$ of $\widetilde{G}$ to be

$$
N= \begin{cases}\left\langle\widetilde{\sigma}_{-1}, \widetilde{\sigma}_{2}, \widetilde{\sigma}_{q}^{2}, \varepsilon\right\rangle & \text { if } p=2, \\ \left\langle\widetilde{\sigma}_{p}, \widetilde{\sigma}_{q}^{2}, \varepsilon\right\rangle & \text { if } p \neq 2 .\end{cases}
$$

It is easy to see that the subgroup $N$ is abelian of index 2 in $\widetilde{G}$ and is a direct sum of the cyclic groups generated by the above elements. Thus we have

$$
N \cong \begin{cases}\mathbb{Z} / 2 \mathbb{Z} \oplus \mathbb{Z} /((q-1) / 2) \mathbb{Z} \oplus \mathbb{Z} / 2 \mathbb{Z} & \text { if } p=-1, \\ \mathbb{Z} / 2 \mathbb{Z} \oplus \mathbb{Z} / 2 \mathbb{Z} \oplus \mathbb{Z} /((q-1) / 2) \mathbb{Z} \oplus \mathbb{Z} / 2 \mathbb{Z} & \text { if } p=2, \\ \mathbb{Z} /(p-1) \mathbb{Z} \oplus \mathbb{Z} /((q-1) / 2) \mathbb{Z} \oplus \mathbb{Z} / 2 \mathbb{Z} & \text { if } p>2 .\end{cases}
$$

In Case B, we define the subgroup $N$ of $\widetilde{G}$ to be

$$
N= \begin{cases}\left\langle\widetilde{\sigma}_{-1}, \widetilde{\sigma}_{2}, \widetilde{\sigma}_{q}^{2}\right\rangle & \text { if } p=2, \\ \left\langle\widetilde{\sigma}_{p}, \widetilde{\sigma}_{q}^{2}\right\rangle & \text { if } p \neq 2 \text { and }\left|\widetilde{\sigma}_{q}\right|=2\left|\sigma_{q}\right|, \\ \left\langle\widetilde{\sigma}_{p}^{2}, \widetilde{\sigma}_{q}\right\rangle & \text { if }\left|\widetilde{\sigma}_{p}\right|=2\left|\sigma_{p}\right| .\end{cases}
$$

Again $N$ is abelian and has index 2 in $\widetilde{G}$. In addition, we have

$$
N \cong \begin{cases}\mathbb{Z} / 2 \mathbb{Z} \oplus \mathbb{Z} / 2 \mathbb{Z} & \text { if }(p, q)=(-1,2), \\ \mathbb{Z} / 2 \mathbb{Z} \oplus \mathbb{Z} /(q-1) \mathbb{Z} & \text { if } p=-1, q>2, \\ \mathbb{Z} / 2 \mathbb{Z} \oplus \mathbb{Z} / 2 \mathbb{Z} \oplus \mathbb{Z} /(q-1) \mathbb{Z} & \text { if } p=2, \\ \mathbb{Z} /(p-1) \mathbb{Z} \oplus \mathbb{Z} /(q-1) \mathbb{Z} & \text { if } p>2 .\end{cases}
$$

In Case C, $p, q$ are both odd prime numbers. Let $v_{2}(p-1)$ denote the power of 2 in $p-1$. We define the subgroup $N$ of $\widetilde{G}$ to be

$$
N= \begin{cases}\left\langle\widetilde{\sigma}_{p}^{2}, \widetilde{\sigma}_{q}\right\rangle & \text { if } v_{2}(p-1) \leq v_{2}(q-1), \\ \left\langle\widetilde{\sigma}_{p}, \widetilde{\sigma}_{q}^{2}\right\rangle & \text { if } v_{2}(p-1)>v_{2}(q-1) .\end{cases}
$$

Then $N$ is an abelian subgroup of $\widetilde{G}$. When $v_{2}(p-1) \leq v_{2}(q-1)$, we have

$$
|N|=\frac{\left|\widetilde{\sigma}_{p}^{2}\right| \cdot\left|\widetilde{\sigma}_{q}\right|}{\left|\left\langle\widetilde{\sigma}_{p}^{2}\right\rangle \cap\left\langle\widetilde{\sigma}_{q}\right\rangle\right|}=\frac{(p-1) \cdot 2(q-1)}{2},
$$


thus $[\widetilde{G}: N]=2$ and $N$ is a normal subgroup of $\widetilde{G}$. We have the same result when $v_{2}(p-1)>v_{2}(q-1)$. Although the subgroup $\left\langle\widetilde{\sigma}_{p}^{2}, \widetilde{\sigma}_{q}\right\rangle$ is always an abelian subgroup of $\widetilde{G}$ of index 2 , when $v_{2}(p-1)>v_{2}(q-1)$ we are not able to get all irreducible representations of $\widetilde{G}$ from this subgroup. So we define $N$ in two cases.

Next we determine the structure of the subgroup $N$ in Case C. We consider the case $v_{2}(p-1) \leq v_{2}(q-1)$ in detail. Let $d=\operatorname{gcd}((p-1) / 2, q-1)$, $s=(p-1) / 2 d$ and $t=(q-1) / d$. Choose $u, v \in \mathbb{Z}$ such that $u s+v t=1$. We have the relations

$$
\left(\widetilde{\sigma}_{p}^{2}\right)^{p-1}=1, \quad\left(\widetilde{\sigma}_{p}^{2}\right)^{(p-1) / 2}=\varepsilon=\widetilde{\sigma}_{q}^{q-1} .
$$

Let $M$ be the free abelian group generated by two words $\alpha, \beta$. Let

$$
\alpha_{1}=(p-1) \alpha, \quad \beta_{1}=\frac{p-1}{2} \alpha-(q-1) \beta,
$$

and let $M_{1}$ be the subgroup of $M$ generated by $\alpha_{1}, \beta_{1}$. Then $M_{1}$ is the kernel of the homomorphism

$$
M \rightarrow N, \quad \alpha \mapsto \widetilde{\sigma}_{p}^{2}, \quad \beta \mapsto \widetilde{\sigma}_{q} .
$$

So we have $N \cong M / M_{1}$. Define the matrix

$$
A=\left(\begin{array}{cc}
p-1 & (p-1) / 2 \\
0 & 1-q
\end{array}\right)
$$

Then $\left(\alpha_{1}, \beta_{1}\right)=(\alpha, \beta) \cdot A$. We determine the structure of $M_{1}$ by considering the standard form of $A$. Define

$$
P=\left(\begin{array}{cc}
u & v \\
-t & s
\end{array}\right) \in \mathrm{SL}_{2}(\mathbb{Z}), \quad Q=\left(\begin{array}{cc}
1 & 2 t v-1 \\
-1 & -2 t v+2
\end{array}\right) \in \mathrm{SL}_{2}(\mathbb{Z}) .
$$

Then

$$
B=P A Q=\left(\begin{array}{cc}
d & 0 \\
0 & -2 s(q-1)
\end{array}\right)
$$

is the standard form of $A$. Let

$$
(\tau, \mu)=(\alpha, \beta) P^{-1} \quad \text { and } \quad\left(\tau_{1}, \mu_{1}\right)=\left(\alpha_{1}, \beta_{1}\right) Q .
$$

Then $\left(\tau_{1}, \mu_{1}\right)=(\tau, \mu) B, M=\mathbb{Z} \tau \oplus \mathbb{Z} \mu$ and $M_{1}=\mathbb{Z} d \tau \oplus \mathbb{Z} 2 s(q-1) \mu$. We thus have

$$
N \cong M / M_{1} \cong \mathbb{Z} / d \mathbb{Z} \oplus \mathbb{Z} / 2 s(q-1) \mathbb{Z} .
$$

By abuse of notation, we also write

$$
(\tau, \mu)=\left(\widetilde{\sigma}_{p}^{2}, \widetilde{\sigma}_{q}\right) P^{-1}=\left(\widetilde{\sigma}_{p}^{2 s} \widetilde{\sigma}_{q}^{t}, \widetilde{\sigma}_{p}^{-2 v} \widetilde{\sigma}_{q}^{u}\right) .
$$

Then $\tau, \mu$ are of order $d, 2 s(q-1)$ respectively, and $N$ is a direct sum of $\langle\tau\rangle$ and $\langle\mu\rangle$. We have $\widetilde{\sigma}_{p}^{2}=\tau^{u} \mu^{-t}$ and $\widetilde{\sigma}_{q}=\tau^{v} \mu^{s}$. When $v_{2}(p-1)>v_{2}(q-1)$, 
we get the structure of $N$ in the same way. So in Case $\mathrm{C}$ we have

$$
N \cong \begin{cases}\mathbb{Z} / d \mathbb{Z} \oplus \mathbb{Z} / 2 s(q-1) \mathbb{Z} & \text { if } v_{2}(p-1) \leq v_{2}(q-1), \\ \mathbb{Z} / d^{\prime} \mathbb{Z} \oplus \mathbb{Z} / 2 s^{\prime}(p-1) \mathbb{Z} & \text { if } v_{2}(p-1)>v_{2}(q-1),\end{cases}
$$

where $d=\operatorname{gcd}((p-1) / 2, q-1), s=(p-1) / 2 d$ and $d^{\prime}=\operatorname{gcd}(p-1,(q-1) / 2)$, $s^{\prime}=(q-1) / 2 d^{\prime}$.

Now we summarize our results in the following

Proposition 2.1. The abelian subgroup $N$ of the group $\widetilde{G}$ of index 2 defined in (A2.1), (B2.1) and (C2.1) has the structure described in (A2.2), $(\mathrm{B} 2.2)$ and $(\mathrm{C} 2.2)$ in Cases $A, B$ and $C$, respectively. In particular, every irreducible representation of $\widetilde{G}$ has dimension 1 or 2 .

3. 2-dimensional representations. We determine all irreducible representations of $\widetilde{G}$ in this section. We will freely use some basic facts from representation theory. For the details, see [5].

It is well-known that the 1-dimensional representations of $\widetilde{G}$ correspond bijectively to those of the maximal abelian quotient $G$ of $\widetilde{G}$, which are Dirichlet characters. So we construct the 2-dimensional irreducible representations of $\widetilde{G}$. From the dimension formula for all irreducible representations, we see that $\widetilde{G}$ has $|G| / 4$ irreducible representations of dimension 2, up to isomorphism. Let $N$ be the subgroup of $\widetilde{G}$ defined in the previous section. Let $\widetilde{G}=N \cup \sigma N$ be the decomposition into cosets. If $\rho: N \rightarrow \mathbb{C}^{*}$ is a representation of $N$, the induced representation $\widetilde{\rho}$ of $\rho$ is a representation of $\widetilde{G}$ of dimension 2. The space of the representation $\widetilde{\rho}$ is $V=\operatorname{Ind}_{N}^{\widetilde{G}}(\mathbb{C})=\mathbb{C}[\widetilde{G}] \otimes_{\mathbb{C}[N]} \mathbb{C}$ with basis $e_{1}=1 \otimes 1$ and $e_{2}=\sigma \otimes 1$. The group homomorphism

$$
\widetilde{\rho}: \widetilde{G} \rightarrow \mathrm{GL}(V) \simeq \mathrm{GL}_{2}(\mathbb{C})
$$

is given by

$$
\widetilde{\rho}(\widetilde{\sigma})=\left(\begin{array}{cc}
\rho(\widetilde{\sigma}) & \rho(\widetilde{\sigma} \sigma) \\
\rho\left(\sigma^{-1} \widetilde{\sigma}\right) & \rho\left(\sigma^{-1} \widetilde{\sigma} \sigma\right)
\end{array}\right), \quad \forall \widetilde{\sigma} \in \widetilde{G},
$$

where $\rho(\widetilde{\sigma})=0$ if $\widetilde{\sigma} \notin N$. The representation $\widetilde{\rho}$ is irreducible if and only if $\rho \not \rho^{\tau}$ for every $\tau \in \widetilde{G} \backslash N$, where $\rho^{\tau}$ is the conjugate representation of $\rho$ defined by

$$
\rho^{\tau}(x)=\rho\left(\tau^{-1} x \tau\right), \quad \forall x \in N .
$$

Since $N$ is abelian, we only need to check $\rho \neq \rho^{\sigma}$.

Now we begin to construct all 2-dimensional irreducible representations of $\widetilde{G}$. As in the previous section, we consider the three cases separately. In addition, we consider the case when $p$ and $q$ are odd prime numbers in detail, and only state the results when $p=-1$ or 2 . 
3.1. Case A. Assume $p>2$. In this case we have $N=\left\langle\widetilde{\sigma}_{p}, \widetilde{\sigma}_{q}^{2}, \varepsilon\right\rangle$ and

$$
N \cong \mathbb{Z} /(p-1) \mathbb{Z} \oplus \mathbb{Z} /((q-1) / 2) \mathbb{Z} \oplus \mathbb{Z} / 2 \mathbb{Z} .
$$

Every irreducible representation of $N$ can be written as $\rho_{i j k}: N \rightarrow \mathbb{C}^{*}$ with

$$
\rho_{i j k}\left(\widetilde{\sigma}_{p}\right)=\zeta_{p-1}^{i}, \quad \rho_{i j k}\left(\widetilde{\sigma}_{q}^{2}\right)=\zeta_{q-1}^{2 j}, \quad \rho_{i j k}(\varepsilon)=(-1)^{k},
$$

where $0 \leq i<p-1,0 \leq j<(q-1) / 2$ and $k=0,1$. Since $\widetilde{G}=N \cup \widetilde{\sigma}_{q} N$ and $\rho_{i j k}^{\widetilde{\sigma}_{q}}\left(\widetilde{\sigma}_{p}\right)=\rho_{i j k}(\varepsilon) \rho_{i j k}\left(\widetilde{\sigma}_{p}\right)=(-1)^{k} \rho_{i j k}\left(\widetilde{\sigma}_{p}\right)$, we have

$$
\rho_{i j k}^{\widetilde{\sigma}_{q}} ¥ \rho_{i j k} \Leftrightarrow k=1 .
$$

Write $\rho_{i j}=\rho_{i j 1}$. The representation $\widetilde{\rho}_{i j}: \widetilde{G} \rightarrow \mathrm{GL}_{2}(\mathbb{C})$ induced from $\rho_{i j}$ is given by

$$
\widetilde{\rho}_{i j}\left(\widetilde{\sigma}_{p}\right)=\left(\begin{array}{cc}
\zeta_{p-1}^{i} & 0 \\
0 & -\zeta_{p-1}^{i}
\end{array}\right), \quad \widetilde{\rho}_{i j}\left(\widetilde{\sigma}_{q}\right)=\left(\begin{array}{cc}
0 & \zeta_{q-1}^{2 j} \\
1 & 0
\end{array}\right), \quad \widetilde{\rho}_{i j}(\varepsilon)=-I,
$$

where $I$ is the identity matrix of degree 2 . Since

$$
\widetilde{\rho}_{i j}\left(\widetilde{\sigma}_{p}^{2}\right)=\left(\begin{array}{cc}
\zeta_{p-1}^{2 i} & 0 \\
0 & \zeta_{p-1}^{2 i}
\end{array}\right) \quad \text { and } \quad \widetilde{\rho}_{i j}\left(\widetilde{\sigma}_{q}^{2}\right)=\left(\begin{array}{cc}
\zeta_{q-1}^{2 j} & 0 \\
0 & \zeta_{q-1}^{2 j}
\end{array}\right),
$$

we see that the representations $\widetilde{\rho}_{i j}$ with $0 \leq i<(p-1) / 2,0 \leq j<(q-1) / 2$ are irreducible and are not isomorphic to each other, by considering the values of the characters of these representations at $\widetilde{\sigma}_{p}^{2}$ and $\tilde{\sigma}_{q}^{2}$. The number of these representations is $\frac{p-1}{2} \cdot \frac{q-1}{2}=\frac{|G|}{4}$. So they are all the irreducible representations of $\widetilde{G}$ of dimension 2 .

Similarly, when $p=-1$, all irreducible representations of $\widetilde{G}$ of dimension 2 are $\widetilde{\rho}_{j}$ with $0 \leq j<(q-1) / 2$, where

$$
\widetilde{\rho}_{j}\left(\widetilde{\sigma}_{-1}\right)=\left(\begin{array}{cc}
1 & 0 \\
0 & -1
\end{array}\right), \quad \widetilde{\rho}_{j}\left(\widetilde{\sigma}_{q}\right)=\left(\begin{array}{cc}
0 & \zeta_{q-1}^{2 j} \\
1 & 0
\end{array}\right), \quad \widetilde{\rho}(\varepsilon)=-I,
$$

and when $p=2$, all irreducible representations of $\widetilde{G}$ of dimension 2 are $\bar{\rho}_{i j}$ with $0 \leq i \leq 1$ and $0 \leq j<(q-1) / 2$, where $\bar{\rho}_{i j}(\varepsilon)=-I$ and

$$
\bar{\rho}_{i j}\left(\widetilde{\sigma}_{-1}\right)=(-1)^{i} I, \quad \bar{\rho}_{i j}\left(\widetilde{\sigma}_{2}\right)=\left(\begin{array}{cc}
1 & 0 \\
0 & -1
\end{array}\right), \quad \bar{\rho}_{i j}\left(\widetilde{\sigma}_{q}\right)=\left(\begin{array}{cc}
0 & \zeta_{q-1}^{2 j} \\
1 & 0
\end{array}\right) .
$$

3.2. Case B. Assume $p>2$ and $\left|\widetilde{\sigma}_{q}\right|=2\left|\sigma_{q}\right|$. Then $N=\left\langle\widetilde{\sigma}_{p}, \widetilde{\sigma}_{q}^{2}\right\rangle$, and

$$
N \cong \mathbb{Z} /(p-1) \mathbb{Z} \oplus \mathbb{Z} /(q-1) \mathbb{Z}
$$

Any irreducible representation of $N$ has the form $\rho_{i j}: N \rightarrow \mathbb{C}^{*}$, where

$$
\rho_{i j}\left(\widetilde{\sigma}_{p}\right)=\zeta_{p-1}^{i}, \quad \rho_{i j}\left(\widetilde{\sigma}_{q}^{2}\right)=\zeta_{q-1}^{j}, \quad \rho_{i j}(\varepsilon)=\rho_{i j}\left(\widetilde{\sigma}_{q}^{2}\right)^{(q-1) / 2}=(-1)^{j},
$$


and $0 \leq i<p-1,0 \leq j<q-1$. It is easy to check that

$$
\rho_{i j}^{\widetilde{\sigma}_{q}} \nRightarrow \rho_{i j} \Leftrightarrow j \equiv 1 \bmod 2 \text {. }
$$

The representation $\widetilde{\rho}_{i j}: \widetilde{G} \rightarrow \mathrm{GL}_{2}(\mathbb{C})$ induced from $\rho_{i j}$ with odd $j$ is given by

$$
\widetilde{\rho}_{i j}\left(\widetilde{\sigma}_{p}\right)=\left(\begin{array}{cc}
\zeta_{p-1}^{i} & 0 \\
0 & -\zeta_{p-1}^{i}
\end{array}\right), \quad \widetilde{\rho}_{i j}\left(\widetilde{\sigma}_{q}\right)=\left(\begin{array}{cc}
0 & \zeta_{q-1}^{j} \\
1 & 0
\end{array}\right)
$$

Since

$$
\widetilde{\rho}_{i j}\left(\widetilde{\sigma}_{p}^{2}\right)=\left(\begin{array}{cc}
\zeta_{p-1}^{2 i} & 0 \\
0 & \zeta_{p-1}^{2 i}
\end{array}\right) \quad \text { and } \quad \widetilde{\rho}_{i j}\left(\widetilde{\sigma}_{q}^{2}\right)=\left(\begin{array}{cc}
\zeta_{q-1}^{j} & 0 \\
0 & \zeta_{q-1}^{j}
\end{array}\right),
$$

we see that the representations $\widetilde{\rho}_{i j}$ with $0 \leq i<(p-1) / 2$ and $0 \leq j<q-1$, $2 \nmid j$ are irreducible and are not isomorphic to each other. The number of these representations is $|G| / 4$. So they are all the irreducible representations of $\widetilde{G}$ of dimension 2 .

Similarly, when $(p, q)=(-1,2)$, there is only one irreducible representation $\widetilde{\rho}_{0}$ of dimension 2 defined by

$$
\widetilde{\rho}_{0}\left(\widetilde{\sigma}_{-1}\right)=\left(\begin{array}{cc}
1 & 0 \\
0 & -1
\end{array}\right), \quad \widetilde{\rho}_{0}\left(\widetilde{\sigma}_{2}\right)=\left(\begin{array}{cc}
0 & -1 \\
1 & 0
\end{array}\right) .
$$

When $p=-1$ and $q>2$, all irreducible representations of dimension 2 are $\widetilde{\rho}_{j}$ with $0 \leq j<q-1,2 \nmid j$, where $\widetilde{\rho}_{j}$ is defined by

$$
\widetilde{\rho}_{j}\left(\widetilde{\sigma}_{-1}\right)=\left(\begin{array}{cc}
1 & 0 \\
0 & -1
\end{array}\right), \quad \widetilde{\rho}_{j}\left(\widetilde{\sigma}_{q}\right)=\left(\begin{array}{cc}
0 & \zeta_{q-1}^{j} \\
1 & 0
\end{array}\right) .
$$

When $p=2$, all irreducible representations of dimension 2 are $\bar{\rho}_{i j}$ with $0 \leq i \leq 1$ and $0 \leq j<q-1,2 \nmid j$, where $\bar{\rho}_{i j}$ is defined by

$$
\bar{\rho}_{i j}\left(\widetilde{\sigma}_{-1}\right)=(-1)^{i} I, \quad \bar{\rho}_{i j}\left(\widetilde{\sigma}_{2}\right)=\left(\begin{array}{cc}
1 & 0 \\
0 & -1
\end{array}\right), \quad \bar{\rho}_{i j}\left(\widetilde{\sigma}_{q}\right)=\left(\begin{array}{cc}
0 & \zeta_{q-1}^{j} \\
1 & 0
\end{array}\right) .
$$

When $\left|\widetilde{\sigma}_{p}\right|=2\left|\sigma_{p}\right|$, all irreducible representations of dimension 2 are $\hat{\rho}_{i j}$ with $0 \leq i<p-1,2 \nmid i$ and $0 \leq j<(q-1) / 2$, where $\hat{\rho}_{i j}$ is defined by

$$
\hat{\rho}_{i j}\left(\widetilde{\sigma}_{p}\right)=\left(\begin{array}{cc}
0 & \zeta_{p-1}^{i} \\
1 & 0
\end{array}\right), \quad \hat{\rho}_{i j}\left(\widetilde{\sigma}_{q}\right)=\left(\begin{array}{cc}
\zeta_{q-1}^{j} & 0 \\
0 & -\zeta_{q-1}^{j}
\end{array}\right) .
$$

3.3. Case C. Assume $v_{2}(p-1) \leq v_{2}(q-1)$. Let

$$
d=\operatorname{gcd}\left(\frac{p-1}{2}, q-1\right), \quad s=\frac{p-1}{2 d}, \quad t=\frac{q-1}{d}, \quad u s+v t=1
$$


as before. Here $t$ must be even and $u$ odd. Let $\tau=\widetilde{\sigma}_{p}^{2 s} \cdot \widetilde{\sigma}_{q}^{t}$ and $\mu=\widetilde{\sigma}_{p}^{-2 v} \cdot \widetilde{\sigma}_{q}^{u}$. Then $N=\left\langle\widetilde{\sigma}_{p}^{2}, \widetilde{\sigma}_{q}\right\rangle=\langle\tau, \mu\rangle$ and

$$
N \cong \mathbb{Z} / d \mathbb{Z} \oplus \mathbb{Z} / 2 s(q-1) \mathbb{Z} .
$$

Any irreducible representation $\rho_{i j}: N \rightarrow \mathbb{C}^{*}$ is of the form

$$
\rho_{i j}(\tau)=\zeta_{d}^{i}=\zeta_{(p-1)(q-1)}^{2 s(q-1) i}, \quad \rho_{i j}(\mu)=\zeta_{2 s(q-1)}^{j}=\zeta_{(p-1)(q-1)}^{d j} .
$$

From $\widetilde{\sigma}_{p}^{2}=\tau^{u} \mu^{-t}$ and $\widetilde{\sigma}_{q}=\tau^{v} \mu^{s}$, we have

$$
\rho_{i j}\left(\widetilde{\sigma}_{p}^{2}\right)=\zeta_{p-1}^{2 s u i-j}, \quad \rho_{i j}\left(\widetilde{\sigma}_{q}\right)=\zeta_{2(q-1)}^{2 t v i+j}, \quad \rho_{i j}(\varepsilon)=\rho_{i j}\left(\widetilde{\sigma}_{p}^{2}\right)^{(p-1) / 2}=(-1)^{j} .
$$

It is easy to show

$$
\rho_{i j}^{\widetilde{\sigma}_{p}} ¥ \rho_{i j} \Leftrightarrow j \equiv 1 \bmod 2 .
$$

The representation $\widetilde{\rho}_{i j}: \widetilde{G} \rightarrow \mathrm{GL}_{2}(\mathbb{C})$ induced from $\rho_{i j}$ with odd $j$ is given by

$$
\tilde{\rho}_{i j}(\tau)=\left(\begin{array}{cc}
\zeta_{d}^{i} & 0 \\
0 & \zeta_{d}^{i}
\end{array}\right), \quad \tilde{\rho}_{i j}(\mu)=\left(\begin{array}{cc}
\zeta_{2 s(q-1)}^{j} & 0 \\
0 & -\zeta_{2 s(q-1)}^{j}
\end{array}\right) .
$$

Here in the first equality we used the fact that $t$ is even, and in the second equality we used the fact that $u$ is odd. Furthermore, we have

$$
\widetilde{\rho}_{i j}\left(\widetilde{\sigma}_{p}\right)=\left(\begin{array}{cc}
0 & \zeta_{p-1}^{2 s u i-j} \\
1 & 0
\end{array}\right), \quad \widetilde{\rho}_{i j}\left(\widetilde{\sigma}_{q}\right)=\left(\begin{array}{cc}
\zeta_{2(q-1)}^{2 t v i+j} & 0 \\
0 & -\zeta_{2(q-1)}^{2 t v i+j}
\end{array}\right) .
$$

By considering the values of the character of $\widetilde{\rho}_{i j}$ at $\tau$ and $\mu^{2}$, we see that all the representations $\widetilde{\rho}_{i j}$ with $0 \leq i<d$ and $0 \leq j<s(q-1), 2 \nmid j$ are irreducible and are not isomorphic to each other. The number of these representations is $d \cdot s(q-1) / 2=|G| / 4$. So they are all the irreducible representations of $\widetilde{G}$ of dimension 2 .

Similarly, if $v_{2}(p-1)>v_{2}(q-1)$, we let

$$
d^{\prime}=\operatorname{gcd}\left(p-1, \frac{q-1}{2}\right), \quad s^{\prime}=\frac{p-1}{d}, \quad t^{\prime}=\frac{q-1}{2 d}, \quad u^{\prime} s^{\prime}+v^{\prime} t^{\prime}=1 .
$$

Then all the irreducible representations of $\widetilde{G}$ of dimension 2 are $\hat{\rho}_{i j}$ with $0 \leq i<d^{\prime}$ and $0 \leq j<t^{\prime}(p-1), 2 \nmid j$, where $\hat{\rho}_{i j}$ is defined by

$$
\hat{\rho}_{i j}\left(\widetilde{\sigma}_{p}\right)=\left(\begin{array}{cc}
\zeta_{2(p-1)}^{2 s^{\prime} u^{\prime} i+j} & 0 \\
0 & -\zeta_{2(p-1)}^{2 s^{\prime} u^{\prime} i+j}
\end{array}\right), \quad \hat{\rho}_{i j}\left(\widetilde{\sigma}_{q}\right)=\left(\begin{array}{cc}
0 & \zeta_{q-1}^{2 t^{\prime} v^{\prime} i-j} \\
1 & 0
\end{array}\right) .
$$

Let $\mathrm{R}^{2}(\widetilde{G})$ be the set of all irreducible representations, up to isomorphism, of $\widetilde{G}$ of dimension 2. To summarize, we have proved the following

THEOREM 3.1. All 2-dimensional irreducible representations of $\widetilde{G}$ are induced from representations of $N$. In detail, we have: 
In Case A

$$
\mathrm{R}^{2}(\widetilde{G})= \begin{cases}\left\{\widetilde{\rho}_{j} \mid 0 \leq j<(q-1) / 2\right\} & \text { if } p=-1, \\ \left\{\bar{\rho}_{i j} \mid i=0,1,0 \leq j<(q-1) / 2\right\} & \text { if } p=2, \\ \left\{\widetilde{\rho}_{i j} \mid 0 \leq i<(p-1) / 2,0 \leq j<(q-1) / 2\right\} & \text { if } p>2,\end{cases}
$$

where $\widetilde{\rho}_{j}, \bar{\rho}_{i j}$ and $\widetilde{\rho}_{i j}$ are defined in (A3.2), (A3.3) and (A3.1) respectively. In Case B

$$
\mathrm{R}^{2}(\widetilde{G})= \begin{cases}\left\{\widetilde{\rho}_{0}\right\} & \text { if }(p, q)=(-1,2), \\ \left\{\widetilde{\rho}_{j} \mid 0 \leq j<q-1,2 \nmid j\right\} & \text { if } p=-1, q>2, \\ \left\{\bar{\rho}_{i j} \mid i=0,1,0 \leq j<q-1,2 \nmid j\right\} & \text { if } p=2, \\ \left\{\hat{\rho}_{i j} \mid 0 \leq i<p-1,2 \nmid i, 0 \leq j<(q-1) / 2\right\} & \text { if }\left|\widetilde{\sigma}_{p}\right|=2\left|\sigma_{p}\right|, \\ \left\{\widetilde{\rho}_{i j} \mid 0 \leq i<(p-1) / 2,0 \leq j<q-1,2 \nmid j\right\} & \text { otherwise, }\end{cases}
$$

where $\widetilde{\rho}_{0}, \widetilde{\rho}_{j}, \bar{\rho}_{i j}, \hat{\rho}_{i j}$ and $\widetilde{\rho}_{i j}$ are defined in (B3.2), (B3.3), (B3.4), (B3.5) and (B3.1) respectively.

In Case $C$

$\mathrm{R}^{2}(\widetilde{G})= \begin{cases}\left\{\widetilde{\rho}_{i j} \mid 0 \leq i<d, 0 \leq j<s(q-1), 2 \nmid j\right\} & \text { if } v_{2}(p-1) \leq v_{2}(q-1), \\ \left\{\hat{\rho}_{i j} \mid 0 \leq i<d^{\prime}, 0 \leq j<t^{\prime}(p-1), 2 \nmid j\right\} & \text { otherwise, }\end{cases}$ where $\widetilde{\rho}_{i j}$ and $\hat{\rho}_{i j}$ are defined in (C3.1) and (C3.2) respectively.

4. The Frobenius maps. This section is a preparation for the next two sections where we will compute the Artin conductors of representations and the Artin $L$-functions of some quasi-cyclotomic fields $\widetilde{K}$. For a prime number $\ell$, we say that $\ell$ is ramified (resp. inert, splitting) in the relative quadratic extension $\widetilde{K} / K$ if the prime ideals of $K$ over $\ell$ are ramified (resp. inert, splitting) in $\widetilde{K}$. For a prime number $\ell$ which is unramified in $\widetilde{K} / K$, let $I_{\ell}\left(\right.$ resp. $\widetilde{I}_{\ell}$ ) be the inert group of $\ell$ in the extension $K / \mathbb{Q}(\operatorname{resp} . \widetilde{K} / \mathbb{Q})$. Let $\mathrm{Fr}_{\ell}$ be the Frobenius automorphism of $\ell$ in $G / I_{\ell}$, and $\widetilde{F r}_{\ell}$ the Frobenius automorphism of $\ell$ in $\widetilde{G} / \widetilde{I}_{\ell}$ associated to some prime ideal over $\ell$.

To compute the Artin conductors of representations, we need to construct a uniformizer in the completion of $\widetilde{K}$ at a prime ideal, in particular at a prime ideal over 2. Generally we are not able to get such a uniformizer, but we can do it in the case $p=-1$. In addition, to calculate the Artin $L$-functions of representations, we need to know $\widetilde{\mathrm{Fr}}_{\ell}$, in particular for $\ell=2$, and so we need to know the decomposition of 2 in $\widetilde{K}$. For odd $p<q \in S$, we calculated some examples by computer which suggest that 2 is always unramified in $\widetilde{K}$. But we are not able to show this. Furthermore, we do not know when 2 splits in $\widetilde{K} / K$ and when 2 is inert in $\widetilde{K} / K$. But when $p=-1$, we can solve these problems (see below). So in this paper we only compute the Artin conductors and Artin $L$-functions of representations in the case $p=-1$. 
From now on, we always assume that $p=-1$, so $K=\mathbb{Q}\left(\zeta_{4 q}\right)$ and $\widetilde{K}=$ $K\left(\sqrt[4]{q^{*}}\right)$. In this section we determine $\widetilde{F r}_{\ell}$ by $\operatorname{Fr}_{\ell}$ for $\ell=2$. In [6, Sect. 5] the decomposition of some odd prime numbers in $\widetilde{K} / K$ was determined. Now we determine the decomposition of 2 in $\widetilde{K} / K$. The result below is a more explicit reformulation of Theorem 2 in [7].

Proposition 4.1. If $q=2$, then 2 is ramified in $\widetilde{K} / K$. If $q$ is odd, then 2 is unramified in $\widetilde{K} / K$ if and only if $\left(\frac{2}{q}\right)=1$, and in this case 2 splits in $\widetilde{K} / K$ if $q^{*} \equiv 1 \bmod 16$, and is inert in $\widetilde{K} / K$ otherwise.

Proof. We first consider the case $q=2$. The unique prime ideal of $K$ over 2 is the principal ideal generated by $\pi_{2}=1-\zeta_{8}$. Since the ramification degree of 2 in $K / \mathbb{Q}$ is 4 and $\sqrt{2}=\pi_{2}\left(\pi_{2}+2 \zeta_{8}\right) \zeta_{8}$, we deduce that 2 is ramified in $\widetilde{K} / K$ if and only if $x^{2} \equiv \sqrt{2} \bmod \pi_{2}^{8}$ is not solvable in the ring $O_{K}$ of integers of $K$ by [7, Th. 2(1)], which is equivalent to $\left(1+\frac{2}{\pi_{2}} \zeta_{8}\right) \zeta_{8}$ not being a square modulo $\pi_{2}^{6}$. Since $2=u \pi_{2}^{4}$ for some unit $u$, we have

$$
\left(1+\frac{2}{\pi_{2}} \zeta_{8}\right) \zeta_{8} \equiv \zeta_{8} \equiv\left(1-\pi_{2}\right) \bmod \pi_{2}^{3},
$$

hence $\left(1+\frac{2}{\pi_{2}} \zeta_{8}\right) \zeta_{8}$ is not a square modulo $\pi_{2}^{3}$. So 2 is ramified in $\widetilde{K} / K$.

Now we assume that $q$ is odd. Let $\pi_{2}=1-\zeta_{4}$. Since the ramification degree of 2 in $K$ is 2 , we see that 2 is unramified in $\widetilde{K} / K$ if and only if $x^{2} \equiv \sqrt{q^{*}} \bmod \pi_{2}^{4}$ is solvable in $O_{K}$ (see [7, Th. 2(1)]). Furthermore, 2 splits in $\widetilde{K} / K$ if and only if $x^{2} \equiv \sqrt{q^{*}} \bmod \pi_{2}^{5}$ is solvable in $O_{K}$. The explicit computation of the Gauss sum gives

$$
\sqrt{q^{*}}=\sum_{a=1}^{q-1}\left(\frac{a}{q}\right) \zeta_{q}^{a}=1+2 \sum_{\left(\frac{a}{q}\right)=1} \zeta_{q}^{a} .
$$

Let $\alpha=\sum_{\left(\frac{a}{q}\right)=1} \zeta_{q}^{a}, \beta=\sum_{\left(\frac{a}{q}\right)=1} \zeta_{2 q}^{a}$, and $\gamma=\sum_{\left(\frac{a}{q}\right)=1} \sum_{\left(\frac{b}{q}\right)=1, a<b} \zeta_{2 q}^{a+b}$, where in the summations $a, b$ run over $1, \ldots, q-1$. Then $\alpha=\beta^{2}-2 \gamma$, which together with the equality $2=\pi_{2}^{2}-\pi_{2}^{3}$ gives

$$
\begin{aligned}
\sqrt{q^{*}} & =1+2 \beta^{2}-4 \gamma=1+\pi_{2}^{2} \beta^{2}-\pi_{2}^{3} \beta^{2}-4 \gamma \\
& \equiv\left(1+\pi_{2} \beta\right)^{2}-\pi_{2}^{3}\left(\beta+\beta^{2}\right)+\pi_{2}^{4}(\beta-\gamma) \\
& \equiv\left(1+\pi_{2} \beta\right)^{2}-\pi_{2}^{3}(\alpha+\beta)+\pi_{2}^{4}(\beta+\gamma) \bmod \pi_{2}^{5} .
\end{aligned}
$$

Since $\zeta_{2 q}=-\zeta_{q}^{-(q-1) / 2}=-\zeta_{q}^{t}$, where $t$ is the inverse of 2 in $(\mathbb{Z} / q \mathbb{Z})^{*}$, we see that $\beta=\sum_{\left(\frac{a}{q}\right)=1}(-1)^{a} \zeta_{q}^{t a} \equiv \sum_{\left(\frac{a}{q}\right)=1} \zeta_{q}^{t a} \bmod 2$. So if $\left(\frac{2}{q}\right)=1$ we have $\alpha \equiv \beta \bmod 2$ and thus 2 is unramified in $\widetilde{K} / K$, and if $\left(\frac{2}{q}\right)=-1$ we have $\alpha+\beta \equiv \sum_{a=1}^{q-1} \zeta_{q}^{a}=-1 \bmod 2$ and thus 2 is ramified in $\widetilde{K} / K$. 
Now we assume $\left(\frac{2}{q}\right)=1$. Then $\sqrt{q^{*}} \bmod \pi_{2}^{5}$ is a square if and only if $\pi_{2} \mid \beta+\gamma$. We consider $2(\beta+\gamma)$. Since $\alpha \equiv \beta \bmod 2$, we have

$$
2(\beta+\gamma)=2 \beta+\beta^{2}-\alpha \equiv \alpha(\alpha+1) \bmod 4 .
$$

From $\sqrt{q^{*}}=1+2 \alpha$, we see that $\alpha(\alpha+1)=\left(q^{*}-1\right) / 4$. Since $8 \mid q^{*}-1$ under the assumption $\left(\frac{2}{q}\right)=1$, we have $\beta+\gamma \equiv\left(q^{*}-1\right) / 8 \bmod 2$. So $\pi_{2} \mid \beta+\gamma$ if and only if $\pi_{2} \mid\left(q^{*}-1\right) / 8$, that is, $2 \mid\left(q^{*}-1\right) / 8$. The proof is complete.

Now we assume that 2 is unramified in $\widetilde{K} / K$. Let $\operatorname{Fr}_{2} \in G$ be such that $\operatorname{Fr}_{2}\left(\zeta_{4}\right)=1$ and $\operatorname{Fr}_{2}\left(\zeta_{q}\right)=\zeta_{q}^{2}$. It is a Frobenius element of 2 in $G$ modulo $I_{2}$. We have $\operatorname{Fr}_{2}=\sigma_{2}^{b_{2}}$ for some $b_{2} \in \mathbb{Z}$ with $2 \mid b_{2}$ as $\left(\frac{2}{q}\right)=1$. Thus $\widetilde{F r}_{2}=\widetilde{\sigma}_{2}^{b_{2}}$ or $\widetilde{\mathrm{Fr}}_{2}=\widetilde{\sigma}_{2}^{b_{2}} \varepsilon$. We need to determine $\widetilde{\mathrm{Fr}}_{2}$ completely. Since $\left(\frac{2}{q}\right)=1$, we have

$$
\sqrt{q^{*}} \equiv\left(1+\pi_{2} \alpha\right)^{2}+\pi_{2}^{4}(\beta+\gamma) \bmod \pi_{2}^{5} .
$$

Write $u=1+\pi_{2} \alpha$ for simplicity. Since $\sqrt{q^{*}} \equiv u^{2} \bmod \pi_{2}^{4}$, we see $\left(\sqrt[4]{q^{*}}-u\right) / 2$ $\in O_{\widetilde{K}}$. Let $\wp$ be the prime ideal of $\widetilde{K}$ over 2 associated to $\widetilde{F r}_{2}$. By the definition, we have

$$
\widetilde{\operatorname{Fr}}_{2}\left(\frac{\sqrt[4]{q^{*}}-u}{2}\right) \equiv\left(\frac{\sqrt[4]{q^{*}}-u}{2}\right)^{2} \equiv(\beta+\gamma)+\frac{\sqrt[4]{q^{*}}-u}{2} \bmod \wp .
$$

On the other hand, since $\widetilde{\sigma}_{q}^{b_{2}}\left(\sqrt[4]{q^{*}}\right)=(-1)^{b_{2} / 2} \sqrt[4]{q^{*}}$ and $\widetilde{\sigma}_{q}^{b_{2}}(u)=u$ as $2 \mid b_{2}$, we have

$$
\widetilde{\sigma}_{q}^{b_{2}}\left(\frac{\sqrt[4]{q^{*}}-u}{2}\right)=\frac{(-1)^{b_{2} / 2} \sqrt[4]{q^{*}}-u}{2}
$$

and

$$
\widetilde{\sigma}_{q}^{b_{2}} \varepsilon\left(\frac{\sqrt[4]{q^{*}}-u}{2}\right)=\frac{(-1)^{b_{2} / 2+1} \sqrt[4]{q^{*}}-u}{2} .
$$

So if $2 \mid b_{2} / 2$ we have $\widetilde{\mathrm{Fr}}_{2}=\widetilde{\sigma}_{2}^{b_{2}}$ if and only if $\pi_{2} \mid \beta+\gamma$ (that is, 2 splits in $\widetilde{K} / K$ ), and if $2 \nmid b_{2} / 2$ we have $\widetilde{F r}_{2}=\widetilde{\sigma}_{2}^{b_{2}}$ if and only if $\pi_{2} \nmid \beta+\gamma$ (that is, 2 is inert in $\widetilde{K} / K)$. In the case $q \equiv 3 \bmod 4$, we can always assume that $2 \nmid b_{2} / 2$, since if $4 \mid b_{2}$, we may replace $b_{2}$ by $b_{2}+(q-1)$. In the case $q \equiv 1 \bmod 4$, we have $2 \mid b_{2} / 2$ iff $2^{(q-1) / 4} \equiv 1 \bmod q$ iff $q$ has the form $A^{2}+64 B^{2}$ for $A, B \in \mathbb{Z}$, by Exercise 28 in Chap. 5 of [4]. So we get the following result:

Proposition 4.2. Assume that 2 is unramified in $\widetilde{K} / K$. Let $\mathrm{Fr}_{2}=\sigma_{2}^{b_{2}}$. Then $2 \mid b_{2}$. If $q \equiv 3 \bmod 4$, we always assume $b_{2} \equiv 2 \bmod 4$. Let $P_{0}$ be the set of prime numbers of the form $A^{2}+64 B^{2}$ with $A, B \in \mathbb{Z}$. Then

$$
\widetilde{\mathrm{F}}_{2}= \begin{cases}\widetilde{\sigma}_{2}^{b_{2}} & \text { if } q \notin P_{0}, 16 \nmid q^{*}-1, \text { or } q \in P_{0}, 16 \mid q^{*}-1, \\ \widetilde{\sigma}_{2}^{b_{2}} \varepsilon & \text { if } q \in P_{0}, 16 \nmid q^{*}-1, \text { or } q \notin P_{0}, 16 \mid q^{*}-1 .\end{cases}
$$

The following lemma is useful in the computation of Artin $L$-functions.

Lemma 4.3. We have $\varepsilon \in \widetilde{I}_{\ell}$ if and only if $\ell$ is ramified in $\widetilde{K} / K$. 
Proof. The canonical projection $\widetilde{G} \rightarrow G \simeq \widetilde{G} /\langle\varepsilon\rangle$ induces a surjective homomorphism $\widetilde{I}_{\ell} \rightarrow I_{\ell}$ which implies the isomorphism $\widetilde{I}_{\ell} /\langle\varepsilon\rangle \cap \widetilde{I}_{\ell} \cong I_{\ell}$. Thus $\ell$ is ramified in $\widetilde{K} / K$ iff $\left|\widetilde{I}_{\ell}\right|=2\left|I_{\ell}\right|$ iff $\left|\widetilde{I}_{\ell} \cap\langle\varepsilon\rangle\right|=2$ iff $\varepsilon \in \widetilde{I}_{\ell}$.

5. The conductors of representations. In this section we compute the Artin conductors of all 2-dimensional irreducible representations of $\widetilde{G}$ in the case $p=-1$. First we recall the definition of the Artin conductor. For details, see [2, Chap. 6].

The notations are as before. Let $\ell$ be a prime number in $\mathbb{Q}$, and choose a prime ideal $\mathfrak{p}$ in $\widetilde{K}$ over $\ell$. Let $\widetilde{G}_{\ell}=\widetilde{G}\left(\widetilde{K}_{\mathfrak{p}} / \mathbb{Q}_{\ell}\right)$ be the corresponding decomposition subgroup. Let $v$ be the normalized valuation in $\widetilde{K}_{\mathfrak{p}}$. For $i \geq 0$, define the ramification groups

$$
\widetilde{G}_{\ell, i}=\left\{\sigma \in \widetilde{G}_{\ell} \mid v(\sigma(x)-x)>i \text { for all } x \in O_{\widetilde{K}_{\mathfrak{p}}}\right\} .
$$

The group $\widetilde{G}_{\ell, 0}$ is the inertia subgroup of $\widetilde{G}_{\ell}$. Let $\pi$ be a uniformizer in $\widetilde{K}_{\mathfrak{p}}$. Then for $i>0$,

$$
\widetilde{G}_{\ell, i}=\left\{\sigma \in \widetilde{G}_{\ell} \mid v(\sigma(\pi)-\pi)>i\right\} .
$$

For a representation $\rho$ of $\widetilde{G}$ with character $\chi$ and representation space $V$, let

$$
f(\chi, \ell)=f(\rho, \ell)=\sum_{i=0}^{\infty} \frac{\left|\widetilde{G}_{\ell, i}\right|}{\left|\widetilde{G}_{\ell, 0}\right|}\left(\chi(1)-\chi\left(\widetilde{G}_{\ell, i}\right)\right),
$$

where $\chi\left(\widetilde{G}_{\ell, i}\right)=\left|\widetilde{G}_{\ell, i}\right|^{-1} \sum_{s \in \widetilde{G}_{\ell, i}} \chi(s)$. We have $f(\chi, \ell)=0$ if $\rho$ is unramified over $\ell$, i.e. $V=V^{\widetilde{G}_{\ell, 0}}$. The Artin conductor of the representation $\rho$ is defined as

$$
\mathfrak{f}(\chi)=\mathfrak{f}(\rho)=\prod_{\ell} \ell^{f(\chi, \ell)} .
$$

From the result in the previous section, we know that $\ell$ is unramified in $\widetilde{K} / \mathbb{Q}$ if $\ell \neq 2, q$. Thus to compute the conductor $\mathfrak{f}(\chi)$, we only need to calculate $f(\chi, 2)$ and $f(\chi, q)$. We consider the cases $q=2$ and $q$ odd separately.

5.1. Case $q=2$. In the case $(p, q)=(-1,2)$, there is only one 2 dimensional irreducible representation $\widetilde{\rho}_{0}$ of $\widetilde{G}$. Let $\widetilde{\chi}_{0}$ be the character of $\widetilde{\rho}_{0}$. Since only 2 is ramified in $\widetilde{K}$, we only need to calculate $f\left(\widetilde{\chi}_{0}, 2\right)$.

As in the previous section, let $\pi_{2}=1-\zeta_{8}$. Let $\wp$ be a prime ideal in $\widetilde{K}$ over 2 and let $v$ be the normalized valuation in $\widetilde{K}_{\wp}$. From the proof of Proposition 4.1 , we see that $\sqrt{2} / \pi_{2}^{2} \equiv 1-\pi_{2} \bmod \pi_{2}^{3}$. Thus

$$
v\left(\frac{\sqrt{2}}{\pi_{2}^{2}}-1\right)=v\left(\frac{\sqrt[4]{2}}{\pi_{2}}-1\right)+v\left(\frac{\sqrt[4]{2}}{\pi_{2}}+1\right)=v\left(\pi_{2}\right)=2 .
$$


We have $v\left(\sqrt[4]{2} / \pi_{2}-1\right)=v\left(\sqrt[4]{2} / \pi_{2}+1\right)=1$. So $\pi=\sqrt[4]{2} / \pi_{2}-1$ is a uniformizer of $\widetilde{K}_{\wp}$. The group $\widetilde{G}$ is generated by $\widetilde{\sigma}_{-1}$ and $\widetilde{\sigma}_{2}$, and $\widetilde{\sigma}_{-1}(\sqrt[4]{2})=\sqrt[4]{2}$ and $\widetilde{\sigma}_{2}(\sqrt[4]{2})=\sqrt[4]{2} / \sqrt{-1}$. Clearly $G_{2,0}=\widetilde{G}$. Furthermore,

$$
\begin{aligned}
v\left(\widetilde{\sigma}_{-1}(\pi)-\pi\right) & =v\left(\frac{\sqrt[4]{2}}{1-\zeta_{8}^{-1}}-\frac{\sqrt[4]{2}}{1-\zeta_{8}}\right)=v\left(-\frac{1+\zeta_{8}}{1-\zeta_{8}} \sqrt[4]{2}\right)=2, \\
v\left(\widetilde{\sigma}_{2}(\pi)-\pi\right) & =v\left(\frac{\sqrt[4]{2} / \sqrt{-1}}{1+\zeta_{8}}-\frac{\sqrt[4]{2}}{1-\zeta_{8}}\right)=v\left(-\frac{1-\zeta_{8}^{3}}{1-\zeta_{8}} \sqrt[4]{2}\right)=2, \\
v(\varepsilon(\pi)-\pi) & =v(-2(\pi+1))=8 .
\end{aligned}
$$

Thus $G_{2,1}=G_{2,0}=\widetilde{G}$ and $G_{2,2}=\cdots=G_{2,7}=\langle\varepsilon\rangle$. By an easy computation we get $\widetilde{\chi}_{0}\left(G_{2,0}\right)=\tilde{\chi}_{0}\left(G_{2,1}\right)=\cdots=\widetilde{\chi}_{0}\left(G_{2,7}\right)=0$, and $\widetilde{\chi}_{0}\left(G_{2, n}\right)=2$ for $n \geq 8$. So we obtain

$$
f\left(\widetilde{\chi}_{0}, 2\right)=2+2+\frac{1}{4} \cdot 2 \cdot 6=7 .
$$

5.2. Case of $q$ odd. To compute $f(\chi, q)$, we consider the cases $\left(\frac{-1}{q}\right)=1$ and $\left(\frac{-1}{q}\right)=-1$ separately. Let $\wp$ be a prime ideal in $\widetilde{K}$ over $q$. Let $v$ be the normalized valuation in $\widetilde{K}_{\wp}$.

5.2.1. Assume $\left(\frac{-1}{q}\right)=1$. Then $q$ is unramified in $\widetilde{K} / K$ but ramified in $K / \mathbb{Q}$. We see $\pi=1-\zeta_{q}$ is a uniformizer of $\widetilde{K}_{\wp}$. Now all 2-dimensional irreducible representations of $\widetilde{G}$ are as in Case A. Let $\widetilde{\chi}_{j}$ be the character of $\widetilde{\rho}_{j}$. It is easy to see that $\widetilde{G}_{q, 0}=\left\langle\widetilde{\sigma}_{q}\right\rangle$. Notice that $\varepsilon \notin \widetilde{G}_{q, 0}$.

Let $1 \neq \widetilde{\sigma} \in \widetilde{G}_{q, 0}$ and $\widetilde{\sigma}\left(\zeta_{q}\right)=\zeta_{q}^{a}, 1<a \leq q-1$. We have

$$
v(\widetilde{\sigma} \pi-\pi)=v\left(\zeta_{q}-\zeta_{q}^{a}\right)=v\left(1-\zeta_{q}^{a-1}\right)=1 .
$$

Thus $\widetilde{G}_{q, n}=\{1\}$ for $n \geq 1$. By an easy computation we get $\widetilde{\chi}_{j}\left(\widetilde{G}_{q, 0}\right)=0$ and $\widetilde{\chi}_{j}\left(\widetilde{G}_{q, n}\right)=2$ for $n \geq 1$. We obtain

$$
f\left(\widetilde{\chi}_{j}, q\right)=2
$$

5.2.2. Assume $\left(\frac{-1}{q}\right)=-1$. Then $q$ is ramified both in $\widetilde{K} / K$ and in $K / \mathbb{Q}$, and all 2-dimensional irreducible representations are as in Case B. Let $\tilde{\chi}_{j}$ be the character of $\tilde{\rho}_{j}$. Since $v\left(1-\zeta_{q}\right)=2$ and $v(\sqrt[4]{-q})=\frac{1}{4}(2(q-1))=$ $(q-1) / 2$, we see that $\pi=\sqrt[4]{-q} /\left(1-\zeta_{q}\right)^{(q-3) / 4}$ is a uniformizer of $q$ in $\widetilde{K}$. It is obvious that $\widetilde{G}_{q, 0}=\left\langle\widetilde{\sigma}_{q}\right\rangle$. Notice that in this case $\varepsilon \in \widetilde{G}_{q, 0}$. 
Let $1 \neq \widetilde{\sigma} \in \widetilde{G}_{q, 0}$ and $\widetilde{\sigma}\left(\zeta_{q}\right)=\zeta_{q}^{a}, 1<a \leq q-1$. We have

$$
\begin{aligned}
v(\widetilde{\sigma} \pi-\pi)+v(\widetilde{\sigma} \varepsilon \pi-\pi) & =v(\widetilde{\sigma} \pi-\pi)+v(-\widetilde{\sigma} \pi-\pi)=v\left(\widetilde{\sigma} \pi^{2}-\pi^{2}\right) \\
& =v\left(\frac{\left(\frac{a}{q}\right) \sqrt{-q}}{\left(1-\zeta_{q}^{a}\right)^{(q-3) / 2}}-\frac{\sqrt{-q}}{\left(1-\zeta_{q}\right)^{(q-3) / 2}}\right) \\
& =v\left(\pi^{2}\right)+v\left(\frac{1-\left(\frac{a}{q}\right)\left(\sum_{i=0}^{a-1} \zeta_{q}^{i}\right)^{(q-3) / 2}}{\left(\sum_{i=0}^{a-1} \zeta_{q}^{i}\right)^{(q-3) / 2}}\right) \\
& =2+v\left(1-\left(\frac{a}{q}\right)\left(\sum_{i=0}^{a-1} \zeta_{q}^{i}\right)^{(q-3) / 2}\right) .
\end{aligned}
$$

Let $t=v\left(1-\left(\frac{a}{q}\right)\left(\sum_{i=0}^{a-1} \zeta_{q}^{i}\right)^{(q-3) / 2}\right)$. We claim that $t=0$. Otherwise $t>0$. Since

$$
\left(\sum_{i=0}^{a-1} \zeta_{q}^{i}\right)^{(q-3) / 2} \equiv a^{(q-3) / 2} \equiv \begin{cases}1 \bmod \left(1-\zeta_{q}\right) & \text { if }\left(\frac{a}{q}\right)=1 \\ -1 \bmod \left(1-\zeta_{q}\right) & \text { if }\left(\frac{a}{q}\right)=-1\end{cases}
$$

we always have $a \equiv 1 \bmod q$ and thus $a=1$, which contradicts the assumption that $a>1$. This shows the claim. Thus $v(\widetilde{\sigma} \pi-\pi)=v(\widetilde{\sigma} \varepsilon \pi-\pi)=1$, as $v(\widetilde{\sigma} \pi-\pi+\widetilde{\sigma} \varepsilon \pi-\pi)=v(2 \pi)=1$. So we get $\widetilde{G}_{q, n}=\{1\}$ for $n \geq 1$. By an easy computation, $\widetilde{\chi}_{j}\left(\widetilde{G}_{q, 0}\right)=0$ and $\widetilde{\chi}_{j}\left(\widetilde{G}_{q, n}\right)=2$ for $n \geq 1$. We obtain

$$
f\left(\widetilde{\chi}_{j}, q\right)=2 \text {. }
$$

Next we compute $f\left(\widetilde{\chi}_{j}, 2\right)$. We consider the cases $\left(\frac{2}{q}\right)=1$ and $\left(\frac{2}{q}\right)=-1$ separately. Let $\wp$ be a prime ideal in $\widetilde{K}$ over 2 . Let $v$ be the normalized valuation in $\widetilde{K}_{\wp}$.

5.2.3. Assume $\left(\frac{2}{q}\right)=1$. Then 2 is unramified in $\widetilde{K} / K$ but ramified in $K / \mathbb{Q}$, and $\pi=1-\zeta_{4}$ is a uniformizer in $\widetilde{K}_{\wp}$. It is easy to see that $\widetilde{G}_{2,0}=\left\langle\widetilde{\sigma}_{-1}\right\rangle$. Notice that in this case $\varepsilon \notin \widetilde{G}_{2,0}$. We have

$$
v\left(\widetilde{\sigma}_{-1} \pi-\pi\right)=v\left(\zeta_{4}-\zeta_{4}^{-1}\right)=v(2)=2 .
$$

Thus $\widetilde{G}_{2,0}=\widetilde{G}_{2,1}=\left\langle\widetilde{\sigma}_{-1}\right\rangle$ and $\widetilde{G}_{2, n}=\{1\}$ for $n>1$. By an easy computation, $\widetilde{\chi}_{j}\left(\widetilde{G}_{2,0}\right)=\widetilde{\chi}_{j}\left(\widetilde{G}_{2,1}\right)=1$ and $\widetilde{\chi}_{j}\left(\widetilde{G}_{2, n}\right)=2$ for $n>1$. We obtain

$$
f\left(\widetilde{\chi}_{j}, 2\right)=1+1=2 .
$$

5.2.4. Assume $\left(\frac{2}{q}\right)=-1$. Now 2 is ramified both in $\widetilde{K} / K$ and in $K / \mathbb{Q}$. As in the previous section, let $\pi_{2}=1-\zeta_{4}, \alpha=\sum_{\left(\frac{a}{q}\right)=1} \zeta_{q}^{a}$ and $\beta=\sum_{\left(\frac{a}{q}\right)=1} \zeta_{2 q}^{a}$, where the summations are over $1 \leq a \leq q-1$. From the previous section we have

$$
\sqrt{q^{*}} \equiv\left(1+\pi_{2} \beta\right)^{2}+\pi_{2}^{3} \bmod \pi_{2}^{4} .
$$


Let $\mu=1+\pi_{2} \beta$. We claim that $\pi=\left(\sqrt[4]{q^{*}}+\mu\right) / \pi_{2}$ is a uniformizer in $\widetilde{K}_{\wp}$. In fact, since

$$
v\left(\sqrt[4]{q^{*}}+\mu\right)+v\left(\sqrt[4]{q^{*}}-\mu\right)=v\left(\sqrt{q^{*}}-\mu^{2}\right)=v\left(\pi_{2}^{3}\right)=6
$$

and $v\left(\left(\sqrt[4]{q^{*}}+\mu\right)+\left(\sqrt[4]{q^{*}}-\mu\right)\right)=v\left(2 \sqrt[4]{q^{*}}\right)=4$, we must have

$$
v\left(\sqrt[4]{q^{*}}+\mu\right)=v\left(\sqrt[4]{q^{*}}-\mu\right)=3
$$

and thus $v\left(\left(\sqrt[4]{q^{*}}+\mu\right) / \pi_{2}\right)=1$.

It is obvious that $\widetilde{G}_{2,0}=\left\{1, \varepsilon, \widetilde{\sigma}_{-1}, \widetilde{\sigma}_{-1} \varepsilon\right\}$. Since $\widetilde{\sigma}_{-1}\left(\sqrt[4]{q^{*}}\right)=\sqrt[4]{q^{*}}$ and $\widetilde{\sigma}_{-1} \varepsilon\left(\sqrt[4]{q^{*}}\right)=-\sqrt[4]{q^{*}}$, we have

$$
\begin{aligned}
v\left(\widetilde{\sigma}_{-1} \pi-\pi\right) & =v\left(\widetilde{\sigma}_{-1} \frac{\sqrt[4]{q^{*}}+1+\pi_{2} \beta}{\pi_{2}}-\frac{\sqrt[4]{q^{*}}+1+\pi_{2} \beta}{\pi_{2}}\right) \\
& =v\left(\widetilde{\sigma}_{-1} \frac{\sqrt[4]{q^{*}}+1}{\pi_{2}}-\frac{\sqrt[4]{q^{*}}+1}{\pi_{2}}\right) \quad\left(\text { since } \widetilde{\sigma}_{-1} \beta=\beta\right) \\
& =v\left(\frac{\sqrt[4]{q^{*}}+1}{1-\zeta_{4}^{-1}}-\frac{\sqrt[4]{q^{*}}+1}{1-\zeta_{4}}\right)=v\left(\sqrt[4]{q^{*}}+1\right) .
\end{aligned}
$$

To compute it, we first claim that $\pi_{2} \nmid \beta$. Otherwise, $2 \mid \beta$ as $\beta \in \mathbb{Q}\left(\zeta_{q}\right)$. From the previous section, we have $\sqrt{q^{*}}=1+2 \alpha$ and $\alpha+\beta \equiv 1 \bmod 2$, thus $\sqrt{q^{*}} \equiv-1+2 \beta \equiv-1 \bmod 4$ and so $q^{*} \equiv 1 \bmod 8$. This contradicts the assumption $\left(\frac{2}{q}\right)=-1$. We have shown the claim. Thus $v(\beta)=0$. Since $v\left(\sqrt[4]{q^{*}}+1+\pi_{2} \beta\right)=3$, we have $v\left(\sqrt[4]{q^{*}}+1\right)=2$, so $v\left(\widetilde{\sigma}_{-1} \pi-\pi\right)=2$.

We now compute $v\left(\widetilde{\sigma}_{-1} \varepsilon \pi-\pi\right)$. We have

$$
\begin{aligned}
v\left(\widetilde{\sigma}_{-1} \varepsilon \pi-\pi\right) & =v\left(\widetilde{\sigma}_{-1} \varepsilon \frac{\sqrt[4]{q^{*}}+1+\pi_{2} \beta}{\pi_{2}}-\frac{\sqrt[4]{q^{*}}+1+\pi_{2} \beta}{\pi_{2}}\right) \\
& =v\left(\frac{-\sqrt[4]{q^{*}}+1}{1-\zeta_{4}^{-1}}-\frac{\sqrt[4]{q^{*}}+1}{1-\zeta_{4}}\right)=v\left(\sqrt[4]{q^{*}}+\zeta_{4}\right) .
\end{aligned}
$$

Observe that

$$
v\left(\sqrt[4]{q^{*}}+\zeta_{4}\right)+v\left(\sqrt[4]{q^{*}}-\zeta_{4}\right)=v\left(\sqrt{q^{*}}+1\right)=v\left(2 \frac{\sqrt{q^{*}}+1}{2}\right)=4,
$$

since $\pi_{2} \nmid\left(\sqrt{q^{*}}+1\right) / 2$. Furthermore, since

$$
v\left(\left(\sqrt[4]{q^{*}}+\zeta_{4}\right)+\left(\sqrt[4]{q^{*}}-\zeta_{4}\right)\right)=v\left(2 \sqrt[4]{q^{*}}\right)=4,
$$

we must have $v\left(\sqrt[4]{q^{*}}+\zeta_{4}\right)=v\left(\sqrt[4]{q^{*}}-\zeta_{4}\right)=2$, so $v\left(\widetilde{\sigma}_{-1} \varepsilon \pi-\pi\right)=2$. In addition, we have

$$
v(\varepsilon \pi-\pi)=v\left(\frac{-\sqrt[4]{q^{*}}+1+\pi_{2} \beta}{\pi_{2}}-\frac{\sqrt[4]{q^{*}}+1+\pi_{2} \beta}{\pi_{2}}\right)=2 .
$$


By the discussion above we have $\widetilde{G}_{2,0}=\widetilde{G}_{2,1}$ and $\widetilde{G}_{2, n}=\{1\}$ for $n>1$. By an easy computation, $\widetilde{\chi}_{j}\left(\widetilde{G}_{2,0}\right)=\widetilde{\chi}_{j}\left(\widetilde{G}_{2,1}\right)=0$ and $\widetilde{\chi}_{j}\left(\widetilde{G}_{2, n}\right)=2$ for $n>2$. We obtain

$$
f\left(\widetilde{\chi}_{j}, 2\right)=2+2=4 .
$$

5.3. Global conductors. By the equalities (5.1)-(5.6) above, we get the following

THEOREM 5.1. In the case $q=2$, the conductor of the unique 2-dimensional irreducible representation $\widetilde{\rho}_{0}$ of $\widetilde{G}$ is equal to $\mathfrak{f}\left(\widetilde{\rho}_{0}\right)=2^{7}$. In the case that $q$ is odd, all the 2-dimensional irreducible representations $\widetilde{\rho_{j}}$ of $\widetilde{G}$ have the conductor $\mathfrak{f}\left({\widetilde{\rho_{j}}}_{j}\right)=2^{2\left(1+\log _{-1}\left(\frac{2}{q}\right)\right)} q^{2}$.

6. The Artin $L$-functions. In this section we compute the Artin $L$ functions of the quasi-cyclotomic fields $\widetilde{K}=\mathbb{Q}\left(\zeta_{4 q}, \sqrt[4]{q^{*}}\right)$.

The $L$-functions associated to the 1-dimensional representations of $\widetilde{G}$ are the well-known Dirichlet $L$-functions. So we compute the $L$-functions associated to the 2-dimensional irreducible representations of $\widetilde{G}$. Let $\varphi: \widetilde{G} \rightarrow$ $\mathrm{GL}(V)$ be a 2-dimensional irreducible representation. The Artin $L$-function $L(\varphi, s)$ associated to $\varphi$ is defined as the product

$$
L(\varphi, s)=\prod_{\ell \text { prime }} L_{\ell}(\varphi, s)
$$

where the local factors are defined as $L_{\ell}(\varphi, s)=\operatorname{det}\left(1-\varphi\left(\widetilde{\operatorname{Fr}}_{\ell}\right) \ell^{-s} \mid V^{\widetilde{I}_{\ell}}\right)^{-1}$. Now we begin to compute them. First we notice that if $\ell$ is ramified in $\widetilde{K} / K$, then $V^{\widetilde{I}_{\ell}}=0$ and $L_{\ell}(\varphi, s)=1$, which is due to the facts that $\varepsilon \in \widetilde{I}_{\ell}$ by Lemma 4.3 and $\varphi(\varepsilon)=-I$ for any irreducible representation $\varphi$ of $\widetilde{G}$ by Theorem 3.1.

6.1. Case $q=2$. By Section 3, there is only one 2-dimensional representation $\widetilde{\rho}_{0}$ in this case, which is defined by

$$
\widetilde{\rho}_{0}\left(\widetilde{\sigma}_{-1}\right)=\left(\begin{array}{cc}
1 & 0 \\
0 & -1
\end{array}\right), \quad \widetilde{\rho}_{0}\left(\widetilde{\sigma}_{2}\right)=\left(\begin{array}{cc}
0 & -1 \\
1 & 0
\end{array}\right) .
$$

Since 2 is ramified in $\widetilde{K} / K$, we have $L_{2}\left(\widetilde{\rho}_{0}, s\right)=1$. Assume that $\ell$ is an odd prime number.

If $\ell \equiv 7 \bmod 8$, then $\operatorname{Fr}_{\ell}=\sigma_{-1}$ and thus $\widetilde{\mathrm{Fr}}_{\ell}=\widetilde{\sigma}_{-1}$ or $\widetilde{\sigma}_{-1} \varepsilon$. In any case we have

$$
L_{\ell}\left(\widetilde{\rho}_{0}, s\right)=\operatorname{det}\left(I \pm\left(\begin{array}{cc}
1 & 0 \\
0 & -1
\end{array}\right) \ell^{-s}\right)^{-1}=\left(1-\ell^{-2 s}\right)^{-1}
$$


If $\ell \equiv 5 \bmod 8$, then $\operatorname{Fr}_{\ell}=\sigma_{2}$ and thus $\widetilde{\mathrm{Fr}}_{\ell}=\widetilde{\sigma}_{2}$ or $\widetilde{\sigma}_{2} \varepsilon$. We have

$$
L_{\ell}\left(\widetilde{\rho}_{0}, s\right)=\operatorname{det}\left(I \pm\left(\begin{array}{cc}
0 & -1 \\
1 & 0
\end{array}\right) \ell^{-s}\right)^{-1}=\left(1+\ell^{-2 s}\right)^{-1} .
$$

If $\ell \equiv 3 \bmod 8$, then $\operatorname{Fr}_{\ell}=\sigma_{-1} \sigma_{2}$ and thus $\widetilde{\mathrm{Fr}}_{\ell}=\widetilde{\sigma}_{-1} \widetilde{\sigma}_{2}$ or $\widetilde{\sigma}_{-1} \widetilde{\sigma}_{2} \varepsilon$. We have

$$
L_{\ell}\left(\widetilde{\rho}_{0}, s\right)=\operatorname{det}\left(I \pm\left(\begin{array}{cc}
1 & 0 \\
0 & -1
\end{array}\right)\left(\begin{array}{cc}
0 & -1 \\
1 & 0
\end{array}\right) \ell^{-s}\right)^{-1}=\left(1-\ell^{-2 s}\right)^{-1} .
$$

If $\ell \equiv 1 \bmod 8$, then $\mathrm{Fr}_{\ell}=1$ and thus $\widetilde{\mathrm{Fr}}_{\ell}=1$ or $\varepsilon$. In this case we must determine $\widetilde{F r}_{\ell}$ completely. Since $\widetilde{\mathrm{Fr}}_{\ell}(\sqrt[4]{2}) \equiv(\sqrt[4]{2})^{\ell} \bmod \wp$ for the prime ideal $\wp$ of $\widetilde{K}$ over $\ell$ associated to $\widetilde{F r}_{\ell}$, we have $\widetilde{F r}_{\ell}=1$ if $2^{(\ell-1) / 4} \equiv 1 \bmod \ell$, and $\widetilde{\mathrm{Fr}}_{\ell}=\varepsilon$ if $2^{(\ell-1) / 4} \equiv-1 \bmod \ell$. As in the previous section, we find that for $\ell \equiv 1 \bmod 8,2^{(\ell-1) / 4} \equiv 1 \bmod \ell$ if and only if $\ell \in P_{0}$. So we have

$$
L_{\ell}\left(\widetilde{\rho}_{0}, s\right)= \begin{cases}\left(1-\ell^{-s}\right)^{-2} & \text { if } \ell \in P_{0} \\ \left(1+\ell^{-s}\right)^{-2} & \text { otherwise. }\end{cases}
$$

We get the Artin $L$-function in the case $(p, q)=(-1,2)$ as follows:

$$
\begin{aligned}
L\left(\widetilde{\rho}_{0}, s\right)= & \prod_{\ell \equiv 3 \text { or } 7 \bmod 8}\left(1-\ell^{-2 s}\right)^{-1} \cdot \prod_{\ell \equiv 5 \bmod 8}\left(1+\ell^{-2 s}\right)^{-1} \\
& \times \prod_{\ell \in P_{0}}\left(1-\ell^{-s}\right)^{-2} \cdot \prod_{\ell \equiv 1 \bmod 8, \ell \notin P_{0}}\left(1+\ell^{-s}\right)^{-2} .
\end{aligned}
$$

6.2. Case of $q$ odd. In this case, all 2-dimensional irreducible representations of $\widetilde{G}$ are $\widetilde{\rho}_{j}$ with $0 \leq j<q-1,2 \mid j$ if $q \equiv 1 \bmod 4$, and $0 \leq j<q-1,2 \nmid j$ if $q \equiv 3 \bmod 4$, where $\widetilde{\rho}_{j}$ is defined by

$$
\widetilde{\rho}_{j}\left(\widetilde{\sigma}_{-1}\right)=\left(\begin{array}{cc}
1 & 0 \\
0 & -1
\end{array}\right), \quad \widetilde{\rho}_{j}\left(\widetilde{\sigma}_{q}\right)=\left(\begin{array}{cc}
0 & \zeta_{q-1}^{j} \\
1 & 0
\end{array}\right), \quad \widetilde{\rho}_{j}(\varepsilon)=-I .
$$

We first determine the local factors $L_{\ell}\left(\widetilde{\rho}_{j}, s\right)$ for $\ell \neq 2, q$. For such $\ell$ we have $V^{\widetilde{I}_{\ell}}=V$. Let $\operatorname{Fr}_{\ell}=\sigma_{-1}^{a_{\ell}} \sigma_{q}^{b_{\ell}}$, which is equivalent to $\ell \equiv(-1)^{a_{\ell}} \bmod 4$ and $\ell \equiv g^{b_{\ell}} \bmod q$, where $g$ is the primitive root $\bmod q$ associated to $\sigma_{q}$. It is easy to compute that

$$
\tilde{\rho}_{j}\left(\widetilde{\sigma}_{q}^{b_{\ell}}\right)=\left(\begin{array}{cc}
0 & \zeta_{q-1}^{j} \\
1 & 0
\end{array}\right)^{b_{\ell}}= \begin{cases}\zeta_{2(q-1)}^{j b_{\ell} I} & \text { if } 2 \mid b_{\ell}, \\
\left(\begin{array}{cc}
0 & \zeta_{2(q-1)}^{j\left(b_{\ell}+1\right)} \\
\zeta_{2(q-1)}^{j\left(b_{\ell}-1\right)} & 0
\end{array}\right) & \text { if } 2 \nmid b_{\ell} .\end{cases}
$$


Furthermore,

$$
\operatorname{det}\left(I-\widetilde{\rho}_{j}\left(\widetilde{\sigma}_{-1}^{a_{\ell}} \widetilde{\sigma}_{q}^{b_{\ell}}\right) \ell^{-s}\right)= \begin{cases}\left(1-\zeta_{2(q-1)}^{j b_{\ell}} \ell^{-s}\right)^{2} & \text { if } a_{\ell}=0,2 \mid b_{\ell}, \\ 1-\zeta_{q-1}^{j b_{\ell} \ell^{-2 s}} & \text { if } a_{\ell}=0,2 \nmid b_{\ell}, \\ 1+\zeta_{q-1}^{j b_{\ell} \ell^{-2 s}} & \text { or } a_{\ell}=1,2 \mid b_{\ell},\end{cases}
$$

and

$$
\operatorname{det}\left(I+\widetilde{\rho}_{j}\left(\widetilde{\sigma}_{-1}^{a_{\ell}} \widetilde{\sigma}_{q}^{b_{\ell}}\right) \ell^{-s}\right)= \begin{cases}\left(1+\zeta_{2(q-1)}^{j b_{\ell}} \ell^{-s}\right)^{2} & \text { if } a_{\ell}=0,2 \mid b_{\ell}, \\ 1-\zeta_{q-1}^{j b_{\ell} \ell^{-2 s}} & \text { if } a_{\ell}=0,2 \nmid b_{\ell}, \\ 1+\zeta_{q-1}^{j b_{\ell} \ell^{-2 s}} & \text { or } a_{\ell}=1,2 \mid b_{\ell}, \\ \text { if } a_{\ell}=1,2 \nmid b_{\ell} .\end{cases}
$$

So we get

$$
L_{\ell}\left(\widetilde{\rho}_{j}, s\right)=\left(1-\zeta_{q-1}^{j b_{\ell}} \ell^{-2 s}\right)^{-1}
$$

if $\ell \equiv 1 \bmod 4$ and $\ell \equiv g^{b_{\ell}} \bmod q$ with $2 \nmid b_{\ell}$, and also if $\ell \equiv 3 \bmod 4$ and $\ell \equiv g^{b_{\ell}} \bmod q$ with $2 \mid b_{\ell}$, while

$$
L_{\ell}\left(\widetilde{\rho}_{j}, s\right)=\left(1+\zeta_{q-1}^{j b_{\ell}} \ell^{-2 s}\right)^{-1}
$$

if $\ell \equiv 3 \bmod 4$ and $\ell \equiv g^{b_{\ell}} \bmod q$ with $2 \nmid b_{\ell}$.

To compute the local factors when $\ell \equiv 1 \bmod 4$ and $\ell \equiv g^{b_{\ell}} \bmod q$ with $2 \mid b_{\ell}$ we must determine $\widetilde{\mathrm{Fr}}_{\ell}$ completely. Since $\left(\frac{\ell}{q}\right)=1$, we have $\left(\frac{q}{\ell}\right)=1$ and $\left(\frac{q^{*}}{\ell}\right)=1$. Let $\alpha_{\ell} \in \mathbb{Z}$ be such that $\alpha_{\ell}^{2} \equiv q^{*} \bmod \ell$. From $\widetilde{\sigma}_{q}^{b_{\ell}}\left(\sqrt[4]{q^{*}}\right)=$ $(-1)^{b_{\ell} / 2} \sqrt[4]{q^{*}}$, we see that $\widetilde{F r}_{\ell}=\widetilde{\sigma}_{q}^{b_{\ell}}$ if $\left(\frac{\alpha_{\ell}}{\ell}\right)=(-1)^{b_{\ell} / 2}$, and $\widetilde{F r}_{\ell}=\widetilde{\sigma}_{q}^{b_{\ell}} \varepsilon$ if $\left(\frac{\alpha_{\ell}}{\ell}\right)=(-1)^{b_{\ell} / 2+1}$. So when $\ell \equiv 1 \bmod 4$ and $\ell \equiv g^{b_{\ell}} \bmod q$ with $2 \mid b_{\ell}$, we have

$$
L_{\ell}\left(\widetilde{\rho}_{j}, s\right)= \begin{cases}\left(1-\zeta_{2(q-1)}^{j b_{\ell}} \ell^{-s}\right)^{-2} & \text { if }\left(\frac{\alpha_{\ell}}{\ell}\right)=(-1)^{b_{\ell} / 2}, \\ \left(1+\zeta_{2(q-1)}^{j b_{\ell}} \ell^{-s}\right)^{-2} & \text { if }\left(\frac{\alpha_{\ell}}{\ell}\right)=(-1)^{b_{\ell} / 2+1} .\end{cases}
$$

Next we compute the local factors $L_{2}\left(\widetilde{\rho}_{j}, s\right)$ and $L_{q}\left(\widetilde{\rho}_{j}, s\right)$. When $\left(\frac{2}{q}\right)=$ -1 , we know from the previous section that 2 is ramified in $\widetilde{K} / K$. So $L_{2}\left(\widetilde{\rho}_{j}, s\right)=1$ in this case. Now we assume $\left(\frac{2}{q}\right)=1$. Since $I_{2}=\left\langle\sigma_{-1}\right\rangle$ and 2 is unramified in $\widetilde{K} / K$, we have $\widetilde{I}_{2}=\left\langle\widetilde{\sigma}_{-1}\right\rangle$ or $\widetilde{I}_{2}=\left\langle\widetilde{\sigma}_{-1} \varepsilon\right\rangle$. The matrices $I+\widetilde{\rho}_{j}\left(\widetilde{\sigma}_{-1}\right)$ and $I+\widetilde{\rho}_{j}\left(\widetilde{\sigma}_{-1} \varepsilon\right)$ have rank 1 , thus $V^{\widetilde{I}_{2}}$ has dimension 1 . Write $\mathrm{Fr}_{2}=\sigma_{2}^{b_{2}}$ with $2 \mid b_{2}$. As in the previous section, we always assume $b_{2} \equiv 2 \bmod 4$ if $q \equiv 3 \bmod 4$. Recall that $P_{0}$ is the set of all prime numbers of the form $A^{2}+64 B^{2}$ with $A, B \in \mathbb{Z}$. Since $\widetilde{\rho}_{j}\left(\widetilde{\sigma}_{2}^{b_{2}}\right)=\zeta_{2(q-1)}^{j b_{2}} I$, by Lemma 4.3 
we have

$$
L_{2}\left(\widetilde{\rho}_{j}, s\right)= \begin{cases}1-\zeta_{2(q-1)}^{j b_{2}} 2^{-s} & \text { if } q \notin P_{0}, 16 \nmid q^{*}-1, \text { or } q \in P_{0}, 16 \mid q^{*}-1, \\ 1+\zeta_{2(q-1)}^{j b_{2}} 2^{-s} & \text { if } q \in P_{0}, 16 \mid q^{*}-1, \text { or } q \notin P_{0}, 16 \mid q^{*}-1 .\end{cases}
$$

When $q \equiv 3 \bmod 4$, we know that $q$ is ramified in $\widetilde{K} / K$. So $L_{q}\left(\widetilde{\rho}_{j}, s\right)=1$ for odd $j$ in this case. Assume $q \equiv 1 \bmod 4$. Since $I_{q}=\left\langle\sigma_{q}\right\rangle$ and $q$ is unramified in $\widetilde{K} / K$, we have $\widetilde{I}_{q}=\left\langle\widetilde{\sigma}_{q}\right\rangle$ or $\widetilde{I}_{2}=\left\langle\widetilde{\sigma}_{q} \varepsilon\right\rangle$. Thus $V^{\widetilde{I}_{q}}=0$ if $j \neq 0$, and $V^{\widetilde{I}_{q}}$ has dimension 1 if $j=0$.

The Frobenius map $\operatorname{Fr}_{q}$ of $q$ in $G$ modulo $I_{q}$ is the identity map. So $\widetilde{F r}_{q}=1$ or $\varepsilon$. In [7, Sect. 5] we have shown that $q$ splits in $\widetilde{K} / K$ if $q \equiv 1 \bmod 8$, and is inert if $q \equiv 5 \bmod 8$. So $\widetilde{\mathrm{Fr}}_{2}=1$ if $q \equiv 1 \bmod 8$, and $\widetilde{\mathrm{Fr}}_{2}=\varepsilon$ if $q \equiv 5 \bmod 8$. Thus we get

$$
L_{q}\left(\widetilde{\rho}_{j}, s\right)= \begin{cases}1 & \text { if } j \neq 0 \\ 1-q^{-s} & \text { if } j=0, q \equiv 1 \bmod 8 \\ 1+q^{-s} & \text { if } j=0, q \equiv 5 \bmod 8\end{cases}
$$

We have computed all the local factors, obtaining

$$
\begin{aligned}
L\left(\widetilde{\rho}_{j}, s\right)= & \left(1-u_{q} \zeta_{2(q-1)}^{j b_{2}} 2^{-s}\right)^{-1}\left(1-(-1)^{(q-1) / 4} q^{-s}\right)^{-\delta_{0 j}} \\
& \times \prod_{\ell \equiv 1,2 \nmid b_{\ell} \text { or } \ell \equiv 3,2 \mid b_{\ell}}\left(1-\zeta_{q-1}^{j b_{\ell}} \ell^{-2 s}\right)^{-1} \\
& \times \prod_{\ell \equiv 3,2 \mid b_{\ell}}\left(1+\zeta_{q-1}^{j b_{\ell}} \ell^{-2 s}\right)^{-1} \prod_{\ell \equiv 1,2 \mid b_{\ell}}\left(1-u_{\ell} \zeta_{2(q-1)}^{j b_{\ell}} \ell^{-s}\right)^{-2},
\end{aligned}
$$

where $u_{q}=1$ if $q \notin P_{0}, 16 \nmid q^{*}-1$ or $q \in P_{0}, 16 \mid q^{*}-1$, and $u_{q}=-1$ otherwise; $\delta_{0 j}=0$ if $j \neq 0$ and $\delta_{00}=1$; and $u_{\ell}=\left(\frac{\alpha_{\ell}}{\ell}\right)(-1)^{b_{\ell} / 2}$. In the above products, "三" denotes congruence modulo 4.

THEOREM 6.1. Except for the Dirichlet L-functions, all Artin L-functions of the Galois extension $\widetilde{K} / \mathbb{Q}$ are explicitly given by (6.1) in the case $q=2$ and by (6.2) in the case of $q$ odd, where in (6.2) $0 \leq j<q-1,2 \mid j$ if $q \equiv 1 \bmod 4$ and $0 \leq j<q-1,2 \nmid j$ if $q \equiv 3 \bmod 4$.

6.3. A formula. Let $\zeta_{\widetilde{K}}(s)$ and $\zeta_{K}(s)$ be the Dedekind zeta functions of $\widetilde{K}$ and $K$ respectively. By Artin's formula for the decomposition of Dedekind zeta functions, we have

$$
\frac{\zeta_{\widetilde{K}}(s)}{\zeta_{K}(s)}=\prod_{\widetilde{\rho}_{j}} \prod_{\ell \text { prime }} L_{\ell}\left(\widetilde{\rho}_{j}, s\right)^{2},
$$

where $\widetilde{\rho}_{j}$ runs over all 2-dimensional irreducible representations of $\widetilde{G}$. When $q=2$, there is only one 2 -dimensional irreducible representation of $\widetilde{G}$. So the 
square of (6.1) gives the formula. When $q$ is odd, by computing $\prod_{\widetilde{\rho}_{j}} L_{\ell}\left(\widetilde{\rho}_{j}, s\right)$, we get the following

COROllary 6.2. For a prime number $\ell \neq q$, let

$$
f_{\ell}=\frac{q-1}{\operatorname{gcd}\left(b_{\ell}, q-1\right)}
$$

be the order of $\ell \bmod q$ and let

$$
g_{\ell}=\operatorname{gcd}\left(b_{\ell}, q-1\right)=\frac{q-1}{f_{\ell}} .
$$

If $q \equiv 1 \bmod 4$, then

$$
\begin{aligned}
\frac{\zeta_{\widetilde{K}}(s)}{\zeta_{K}(s)}= & \left(1-u_{q}^{f_{2}} 2^{-f_{2} s}\right)^{-g_{2}}\left(1-(-1)^{(q-1) / 4} q^{-s}\right)^{-2} \prod_{\ell \equiv 1,2 \nmid b_{\ell} \text { or } \ell \equiv 3}\left(1-\ell^{-f_{\ell} s}\right)^{-2 g_{\ell}} \\
& \times \prod_{\ell \equiv 1,2 \mid b_{\ell}}\left(1-u_{\ell}^{f_{\ell}} \ell^{-f_{\ell} s}\right)^{-2 g_{\ell}}
\end{aligned}
$$

and if $q \equiv 3 \bmod 4$, then

$$
\begin{aligned}
\frac{\zeta_{\widetilde{K}}(s)}{\zeta_{K}(s)}= & \left(1+u_{q}^{f_{2}} 2^{-f_{2} s}\right)^{-g_{2}} \prod_{\ell \equiv 1,2 \nmid b \ell}\left(1+\ell^{-f_{\ell} s}\right)^{-2 g_{\ell}} \prod_{\ell \equiv 3}\left(1-\ell^{-2 f_{\ell} s}\right)^{-g_{\ell}} \\
& \times \prod_{\ell \equiv 1,2 \mid b_{\ell}}\left(1+u_{\ell}^{f_{\ell}} \ell^{-f_{\ell} s}\right)^{-2 g_{\ell}},
\end{aligned}
$$

where $u_{q}$ and $u_{\ell}$ are as above.

6.4. The corresponding modular forms. All the 2-dimensional irreducible representations of $\widetilde{G}$ in the case $p=-1$ are monomial. It is easy to see that they are odd. By Deligne-Serre's theorem [6, Th. 2], these Artin $L$-functions above are equal to the $L$-functions of some normalized newforms of weight one, which allows one to determine a newform of weight one from a 2-dimensional irreducible odd representation of $\widetilde{G}$. More precisely, the irreducible representation $\widetilde{\rho}_{j}$ of conductor $N$ corresponds to a normalized newform $f_{j}(z)$ of weight one on $\Gamma_{0}(N)$ with nebentype $\phi_{j}=\operatorname{det}\left(\widetilde{\rho}_{j}\right)$, which has a Fourier expansion at infinity

$$
f_{j}(z)=\sum_{n=1}^{\infty} a_{n}^{(j)} q^{n}, \quad q=e^{2 \pi i z},
$$

where $a_{1}^{(j)}=1$ and the other coefficients $a_{n}$ are equal to those of the $L$ function $L\left(\phi_{j}, s\right)=\sum_{n=1}^{\infty} a_{n} n^{-s}$. In this subsection we describe these modular forms explicitly. Since these newforms are eigenfunctions of Hecke operators, to determine all $a_{n}^{(j)}$ it is enough to determine $a_{\ell}^{(j)}$ for all primes $\ell$.

When $q=2$, we get one normalized newform $f_{0}(z)$ of weight 1 on $\Gamma_{0}\left(2^{7}\right)$ with nebentype $\phi_{0}:(\mathbb{Z} / 8 \mathbb{Z})^{*} \rightarrow \mathbb{C}^{*}$, where $\phi_{0}\left(\sigma_{-1}\right)=-1$ and $\phi_{0}\left(\sigma_{2}\right)=1$. 
By (6.1), we directly see that for primes $\ell$ the coefficients $a_{\ell}^{(0)}$ of the newform are given by

$$
a_{\ell}^{(0)}= \begin{cases}0 & \text { if } \ell=2 \text { or } \ell \equiv 3,5,7 \bmod 8, \\ 2 & \text { if } \ell \in P_{0}, \\ -2 & \text { if } \ell \equiv 1 \bmod 8 \text { but } \ell \notin P_{0} .\end{cases}
$$

When $q$ is odd, we get $(q-1) / 2$ normalized newforms $f_{j}(z)$ of weight 1 on $\Gamma_{0}\left(4^{1+\log _{-1}\left(\frac{2}{q}\right)} q^{2}\right)$ with nebentype $\phi_{j}:(\mathbb{Z} / 4 q \mathbb{Z})^{*} \rightarrow \mathbb{C}^{*}$, where $\phi_{j}\left(\sigma_{-1}\right)=-1$ and $\phi_{j}\left(\sigma_{q}\right)=-\zeta_{q-1}^{j}$. By (6.2) we directly see that for primes $\ell \neq q$ the coefficients of the newforms are given by

$$
a_{\ell}^{(j)}= \begin{cases}u_{q} \zeta_{2(q-1)}^{j b_{2}} & \text { if } \ell=2 \\ 2 u_{\ell} \zeta_{2(q-1)}^{j b_{\ell}} & \text { if } \ell \equiv 1 \bmod 4 \text { and } 2 \mid b_{\ell} \\ 0 & \text { otherwise }\end{cases}
$$

and

$$
a_{q}^{(j)}= \begin{cases}0 & \text { if } j \neq 0, \\ (-1)^{(q-1) / 4} & \text { if } j=0,\end{cases}
$$

where $0 \leq j<q-1,2 \mid j$ if $q \equiv 1 \bmod 4$, and $0 \leq j<q-1,2 \nmid j$ if $q \equiv 3 \bmod 4 ; b_{\ell}$ is defined by $\ell \equiv g^{b_{\ell}} \bmod q$ for a primitive root $g$ modulo $q$; $u_{\ell}=\left(\frac{\alpha_{\ell}}{\ell}\right)(-1)^{b_{\ell} / 2}$ for an integer $\alpha_{\ell}$ such that $\alpha_{\ell}^{2} \equiv q^{*} \bmod \ell$; and

$$
u_{q}= \begin{cases}1 & \text { if } q \notin P_{0}, 16 \nmid q^{*}-1 \text { or } q \in P_{0}, 16 \mid q^{*}-1, \\ -1 & \text { if } q \notin P_{0}, 16 \mid q^{*}-1 \text { or } q \in P_{0}, 16 \nmid q^{*}-1 .\end{cases}
$$

Here $P_{0}$ is the set of all primes of the form $A^{2}+64 B^{2}$ with $A, B \in \mathbb{Z}$.

Acknowledgments. The authors thank the referee for the careful reading of the manuscript and for correcting some misprints.

S. Bae is supported by NRF of Korea (ASARC R11-2007-035-01001-0). L. S. Yin is supported by NSFC (No. 10871107).

\section{References}

[1] G. W. Anderson, Kronecker-Weber plus epsilon, Duke Math. J. 114 (2002), 439-475.

[2] J. W. S. Cassels and A. Fröhlich (eds.), Algebraic Number Theory, Academic Press, 1967.

[3] P. Das, Algebraic Gamma monomials and double coverings of cyclotomic fields, Trans. Amer. Math. Soc. 352 (2000), 3557-3594.

[4] K. Ireland and M. Rosen, A Classical Introduction to Modern Number Theory, Grad. Texts in Math. 84, Springer, 1982.

[5] J.-P. Serre, Linear Representations of Finite Groups, Grad. Texts in Math. 42, Springer, New York, 1977.

[6] -, Modular forms of weight one and Galois representations, in: Algebraic Number Fields: L-functions and Galois Properties, A. Fröhlich (ed.), Academic Press, London, 1977, 193-268. 
[7] L. S. Yin and C. Zhang, Arithmetic of quasi-cyclotomic fields, J. Number Theory 128 (2008), 1717-1730.

[8] L. S. Yin and Q. Z. Zhang, All double coverings of cyclotomic fields, Math. Z. 253 (2006), 479-488.

Sunghan Bae

Department of Mathematics

KAIST

Daejeon, Korea
Yong $\mathrm{Hu}$, Linsheng Yin (corresponding author)

Department of Mathematical Sciences

Tsinghua University

Beijing 100084, China

E-mail: lsyin@math.tsinghua.edu.cn

Received on 24.3.2009

and in revised form on 6.9.2009 